\title{
Estructura óptima de capital para empresas en mercados maduros de economías emergentes: una aplicación *
}

\author{
Optimal Capital Structure for an Emerging \\ Market Firm in a Mature Business Sector
}

Estrutura ótima de capital para empresas em mercados maduros de economias emergentes: uma aplicação

\section{Hernán Herrera-Echeverri ${ }^{* \star}$}

Fecha de recibido: 7 de marzo de 2017

Fecha de aprobado: 26 de julio de 2017

Doi: http://dx.doi.org/10.12804/revistas.urosario.edu.co/empresa/a.5463

Para citar: Herrera-Echeverri, H. (2018). Estructura óptima de capital para empresas en mercados maduros de economías emergentes: una aplicación. Universidad \& Empresa, 20(34), 157-191. Doi: http://dx.doi. org/10.12804/revistas.urosario.edu.co/empresa/a.5463

\section{RESUMEN}

En este trabajo, se utiliza el criterio de costo mínimo para determinar la estructura óptima de capital de una empresa del sector de procesamiento de alimentos en un mercado emergente, en este caso, el colombiano. Se abordan y armonizan varios aspectos relacionados

\footnotetext{
Este artículo es preparado únicamente con fines de discusión académica y usa supuestos propios, no oficiales de proyección, e información parcial de carácter público de la empresa que participó en la investigación (se reserva aquí su nombre por fines de confidencialidad). El autor no conoce ni ha estado presentes en la empresa. El caso presenta un análisis que pretende crear un espacio activo de discusión para que estudiosos aborden una comprensión aplicada de la teoría de estructura de capital. El caso no pretende ser una fuente primaria de datos, ratificación o ilustración de una gerencia efectiva o inefectiva.

** Profesor Asociado, Director Maestría en Administración Financiera, Escuela de Economía y Finanzas, Universidad EAFIT. Correo electrónico: hherrer2@eafit.edu.co
} 
con la política de endeudamiento para demostar que el ratio óptimo de deuda maximiza simultáneamente el valor de la empresa y de las acciones, pero, a su vez, minimiza el costo promedio ponderado de capital (Wacc). Mediante el cálculo recursivo se determina el valor de mercado del capital propio, basado en los fundamentales de la empresa y se hace un contraste con el valor pactado en una transacción de mercado reciente. El caso permite examinar algunos de los mecanismos asociados a la teoría de la estructura de capital y aterrizarlos a resultados concretos en una empresa real, para así proponer una reestructuración del nivel de endeudamiento.

Palabras clave: Estructura de capital, costo de capital, endeudamiento.

\section{ABSTRACT}

In this work, minimum cost criteria are used to determine the optimal capital structure for a firm in the food processing industry of an emerging market (Colombia). It is an excellent opportunity to examine how the components of the firm's financial statements interact to calculate optimal capital structure. Several issues related to debt policy are addressed and harmonized to demonstrate that optimal debt ratio simultaneously maximizes the enterprise and share value, and minimizes the weighted average cost of capital (WACC). Recursive calculation is used to establish equity value using the firm's fundamentals, and it is compared to a recent market transaction. This case permits an observation of the mechanisms associated with capital structure theory, and implements them through concrete results in a real-world firm, enabling analysts to propose a level of indebtedness restructuring.

Keywords: Capital structure, cost of capital, indebtedness.

\section{RESUMO}

Neste trabalho, utiliza-se o critério de custo mínimo para determinar a estrutura ótima de capital de uma empresa do setor de processamento de alimentos em um mercado emergente (Colômbia). Se abordam e harmonizam vários aspectos relacionados com a política de endividamento para demonstrar que o rácio ótimo da dívida simultaneamente, maximiza o valor da empresa e das ações, mas para além minimiza o custo médio ponderado de capital (Wacc). Mediante o cálculo recursivo determina-se o valor de mercado do capital próprio, baseado nos fundamentos da empresa e se faz um contraste com o valor pactuado em uma transação de mercado recente. $\mathrm{O}$ caso permite examinar alguns dos mecanismos associados à teoria da estrutura de capital e aterra-los a resultados concretos em uma empresa real, para propor uma restruturação do nível de endividamento.

Palavras-chave: Estrutura de capital, custo de capital, endividamento. 


\section{INTRODUCCIÓN}

Hay una serie de elecciones relacionadas con la determinación de la estructura de capital de una empresa; entre las más comunes están aquellas asociadas con definir la mezcla entre deuda y capital propio y seleccionar los instrumentos de deuda adecuados, con su estructura de pago, costo y tiempo de maduración. Esta decisión, también incluye establecer cómo la empresa distribuirá el riesgo y los derechos sobre los flujos de caja y/o los derechos de voto entre sus proveedores de capital. Además, se deben considerar diferentes tipos de emisión de capital propio, que incluyen instrumentos híbridos con tipos particulares de derechos de propiedad, tales como bonos convertibles y acciones preferentes o sustitutos de la deuda, como los diferentes tipos de leasing.

No hay consenso en la literatura acerca de cómo seleccionar la estructura óptima de capital para una empresa, $\mathrm{y}$ aunque existen varias teorías al respecto, se duda de la aplicación práctica de muchas de ellas a los mercados emergentes, debido a las característi- cas de riesgo, liquidez, tamaño, disponibilidad de información y acceso a fuentes de financiación presentes en este tipo de mercados. En este trabajo se usan varias técnicas que permiten aplicar algunos de tales conceptos a una empresa particular del sector de alimentos en una economía emergente como la colombiana.

Para esos efectos, se utiliza el Capital Asset Pricing Model (CAPM) y el ranking sintético de cobertura con el propósito de estimar el costo de la deuda y determinar el apalancamiento óptimo que equilibra los beneficios y costos financieros marginales del endeudamiento, los cuales se relacionan con el incremento de las tasas de interés por parte de los proveedores de deuda, cuando perciben mayor probabilidad de no pago generada por una baja cobertura de intereses. ${ }^{1}$ Además, se evidencia que en la medida que aumenta el endeudamiento se incrementa el riesgo, y los inversionistas incrementarán sus expectativas de rendimiento. También, se denota que la estructura de capital óptima maximiza simultáneamente el valor de la firma y el precio de la participación

1 En este trabajo se calcula el umbral entre los beneficios del escudo fiscal y los costos adicionales por incremento de las tasas que imponen las organizaciones de crédito cuando los niveles de cobertura indican riesgo de no pago. Sin embargo, los costos de insolvencia financiera se pueden reflejar en muchos otros factores de costo adicional de orden comercial, operacional, reputacional, etc., que afectan la continuidad de la empresa. Una mayor descripción puede obtenerse en: Almeida y Philippon (2008), Bris, Welch y Zhu (2006), Glover, (2016), Hakkio, C. y Keeton, W. (2009), Lian (2017). 
de cada socio (acciones o participación societaria), a su vez que minimiza el costo promedio ponderado del capital.

La empresa en estudio realizó una emisión de bonos para reconvertir su deuda bancaria previamente; no obstante, durante 2014 y 2015 tuvo que obtener una nueva deuda para realizar inversiones adicionales, cuyos resultados se verían a partir de 2016. Aunque el ingreso durante 2015 solo creció el 7\%, las proyecciones de la empresa pronostican que su flujo de caja operacional en 2016 crecerá de manera significativa por dos motivos, el incremento en la eficiencia operacional y en las ventas, los cuales serían resultados de las inversiones hechas durante los años previos. Bajo este nuevo escenario se analiza la estructura actual del capital y se proponen opciones de mejoramiento.

En la primera sección de este artículo se hace una breve revisión de la literatura. En la segunda, se provee la información básica de la empresa. Después, se usa esta información y otra adicional del mercado, para determinar la estructura óptima de capi- tal usando el criterio del menor costo. Al final, se analizan los resultados y se exponen breves conclusiones.

El análisis de resultados se realizó de la siguiente manera. En primer lugar, se contrastó el óptimo hallado con la situación actual de la compañía; luego, se verificó el costo de la deuda versus el costo teórico, según sus niveles de cobertura actual, y después, se calculó el costo de capital que deberían exigir los inversionistas de acuerdo con el nivel de endeudamiento. En adición a lo anterior, y debido a que la empresa hizo recientemente una emisión de acciones privilegiadas, se determinó cuál debería ser el crecimiento implícito a perpetuidad para que los fundamentales coincidan con el valor transado; este último ejercicio se hizo con fines académicos exclusivamente. Al final, se estableció el valor generado por la estructura óptima propuesta y se hizo una propuesta de recapitalización para incrementar el valor da las acciones.

\section{REVISIÓN DE LA LITERATURA²}

Aunque el tema ha sido tratado durante varios años por los investiga-

2 No pretende ser un ejercicio exhaustivo, solo se mencionan algunos trabajos útiles para contextualizar el caso. Una revisión más amplia puede hallarse en: Harris y Raviv (1991), Graham y Leary (2011), Parsons y Titman (2008), Meyers (1977, 1984), Baker y Wurgler (2002), Brennan y Schwartz (1984), Popescu y Visinescu (2009), Rajan y Zingales (1995), Vasiliou y Daskalakis (2009), Barclay, Smith y Watts (1995), Graham (2000, 2003), Hovakimian, Opler y Titman (2001), Leland (1998), Titman y Wessels (1988). 
dores más importantes del área financiera, no existe un consenso en la literatura acerca de cómo conformar la estructura óptima de capital. El trabajo seminal de Modigliani y Miller (1958) menciona que la estructura de capital no tiene implicaciones sobre el valor de la empresa en un mundo de información perfecta sin impuestos, ni costos de transacción, en donde todos los tomadores de decisiones comparten el objetivo de maximizar el valor para los inversionistas; desde ese punto inicial, la teoría de estructura de capital ha evolucionado tratando de relajar varios de esos supuestos.

Miller (1977) indica que en un mundo con impuestos, una firma puede encontrar su estructura de capital óptima en el umbral entre los ahorros que se generan por el escudo fiscal del pago de intereses y el estrés financiero asociado a bajos niveles de cobertura, que incrementan la posibilidad de no pago. Por su parte, algunas investigaciones han entregado una mirada más cercana a las bases que constituyen los costos de estrés financiero (Titman, 1984; Shleifer \& Vishny, 1992). Aunque este enfoque es uno de los más utilizados hoy, no pone atención sobre aspectos fundamentales asociados a la estructura de capital, por ejemplo, cómo debe hacerse la localización de los derechos de control sobre las decisiones y los flujos de caja, o si el capital propio se obtiene interna o externamente. Más aún, este enfoque deja por fuera el hecho de que algunas veces ciertos jugadores pueden tener más información que otros y su propia agenda, la cual no necesariamente coincide con el objetivo de incrementar el valor de la firma.

Recientes contribuciones que abordan los problemas mencionados arriba vienen desde la teoría de agencia, la cual se enfoca en los incentivos que las partes (gerentes e inversionistas) tienen para asumir un comportamiento particular (Jensen \& Meckling, 1976). Jensen (1986) sugiere que altos niveles de deuda imponen disciplina sobre los gerentes para alcanzar resultados y ayudan a reducir los costos de agencia de la financiación con capital propio. Por otro lado, los costos de agencia de la deuda (Myers, 1977; Harris \& Raviv, 1991) se pueden presentar cuando un incremento no adecuado del endeudamiento limita la posibilidad de que la empresa pueda acometer proyectos altamente rentables (underinvestments) o tal endeudamiento sea dirigido para financiar proyectos de excesivo riesgo (overinvestments).

Otros problemas de agencia se presentan debido a la asimetría de la información (los gerentes tienen más 
información que los inversionistas). La información imperfecta en los mercados incrementa el costo de conseguir capital, y quienes poseen información precisa sobre la empresa (insiders) toman decisiones para enviar señales al mercado que permitan a los inversionistas (outsiders) tener confirmaciones sobre el estado actual y las proyecciones futuras de las empresas; por tanto, tales señales afectan el valor de las firmas (Ross, 1997).

Por ejemplo, cuando las firmas buscan nuevos socios, los interesados deben establecer si la necesidad de capital corresponde a la oportunidad de acometer proyectos rentables (buenas noticias) o si se trata de que los gerentes están actuando de manera oportunista, porque el mercado está sobrevalorando el potencial real de la compañía (malas noticias). A menos que los gerentes puedan enviar rápidamente una señal al mercado indicando claramente que se trata de buenas noticias, es probable que el valor de la empresa caiga.

Por otro lado, cuando las empresas buscan y conceden contratos de deuda, ello no implica necesariamente un efecto negativo sobre el valor de la compañía. Generalmente, el mercado asocia la deuda con buenas noticias (Myers \& Majluf, 1984; Smith, 1986), porque los pro- veedores de deuda buscan buenas garantías (análisis de flujo de caja futuro y colaterales) y saben que si hay problemas reales, su pago solo será afectado si esas estas fallan, por lo tanto, la pérdida esperada se reduce por debajo de la pérdida de los dueños.

Myers (1984) ha propuesto la teoría del Pecking order de la estructura de capital, basada en el hecho de que la asimetría de información incrementa los costos de conseguir capital. Esta teoría indica que las empresas buscarán financiar internamente sus nuevos proyectos, hasta el punto que tales recursos les alcancen. Después de agotarse la generación interna de fondos, las empresas preferirán suscribir nueva deuda y solo cuando sea absoluta y estrictamente necesario, buscarán emitir nuevo capital. La explicación de este orden de preferencia obedece a la minimización de los costos de transacción, muchos de ellos generados por la asimetría de información mencionada antes. Esta teoría tiene entonces dos implicaciones básicas: la primera es que las empresas pueden no tener un objetivo de mezcla entre deuda y capital propio en el largo plazo, solo ajustan la participación de cada fuente de recursos en sus estados financieros, dependiendo de los fondos generados y de las oportunidades de inversión que surjan 
en cada período; la segunda es que esta teoría explica una de las causas por las cuales los gerentes prefieren mantener excesos de caja.

El presente trabajo se concentra en determinar la estructura óptima de capital, bajo el criterio de costo mínimo, en un mercado emergente para una empresa dentro de un sector industrial maduro. El apalancamiento óptimo es determinado por el equilibrio entre los beneficios y los costos marginales del endeudamiento. Este caso constituye una aplicación de la literatura revisada a una empresa en concreto, con el fin de proponer una reestructuración deuda-capital propio, que maximice el valor de la empresa y de las acciones, pero que al mismo tiempo minimice el costo de capital de la empresa.

\section{DESCRIPCIÓN DE LA EMPRESA}

La empresa Alimentos Blancos $(\mathrm{AB})^{3}$ produce leche y sus derivados. Los productos más vendidos incluyen una gran variedad de tipos de leche de ganado vacuno: entera, enriquecida, deslactosada, semidescremada y saborizada, entre otras. También produce otros lácteos fermentados, como yogures, kumis, crema de leche, quesos de diferentes variedades y mantequilla. Recientemente, ha impulsado una nueva categoría en su portafolio que incluye una gran variedad de postres y dulces. A pesar de no ser derivados de la leche, la empresa se ha diversificado hacia las bebidas refrescantes y néctares de fruta, diferenciado estos últimos porque son enriquecidos con minerales y vitaminas.

Desde sus inicios, $\mathrm{AB}$ se ha comprometido con ofrecer al consumidor productos de alta calidad para diferentes segmentos; por ejemplo, cuenta con la línea infantil y la línea dietética, que tiene productos bajos en grasa y reducidos en calorías. $\mathrm{AB}$ ha alcanzado importantes niveles de desarrollo de producto y ofrece alimentos funcionales que, además de cumplir su función nutritiva, cuentan con beneficios a nivel gastrointestinal y fortalecen el sistema inmune.

$\mathrm{AB}$ nació en los cuarenta como un fabricante artesanal de quesos, que en la década siguiente construyó su primera planta de producción industrial. Desde esa época, se ha destacado por ser una empresa con la más excelsa cultura de calidad, confianza e innovación; esto los ha

3 Nombre ficticio. Todo el contenido de esta sección está basado en información pública leída de la página web de la empresa. El nombre es obviado con fines de confidencialidad. 
llevado a conservar una alta satisfacción del cliente con productos de gran beneficio para el consumidor. En los años setenta dejó de ser una empresa familiar y diversificó sus derechos de propiedad, incluyendo nuevos accionistas; posteriormente, inició sus primeros procesos de expansión y crecimiento no orgánico durante la década de los ochenta.

Ya entrados los 2000, la compañía logra un crecimiento importante en geografías y unidades de negocio (las ventas consolidadas en 2015 ascienden a 560 millones de dólares); por tal motivo, desarrolla un esquema corporativo de operación implementando su Centro de Servicios Compartidos (ASC) y la integración de la cadena de logística. También, consolida la expansión internacional, principalmente en Estados Unidos, y continúa destacándose por su caracter innovador al crear un instituto dedicado a la investigación en alimentación y nutrición. Otro hito a resaltar es la consolidación de su compromiso con el medioambiente y su alto sentido social mediante la creación de su Fundación, que es una muestra contundente de su responsabilidad social empresarial.
Para financiar este crecimiento, en 2009 se diseñó un cambio en el modelo de endeudamiento, a través de la estructuración de bonos a largo plazo. $\mathrm{AB}$ emitió bonos por 89,7 millones de dólares al mercado Colombiano. Durante 2014 y 2015 fue necesario adquirir una nueva deuda para realizar inversiones adicionales, cuyos resultados se verían a partir de 2016. Aunque el ingreso durante 2015 creció el 7\%, las proyecciones de la empresa pronosticaban que su flujo de caja operacional en 2016 crecería de manera significativa por dos motivos: el incremento en la eficiencia operacional y en las ventas, ambos resultado de las inversiones hechas durante los años previos, gracias a la estrategia de sostenibilidad que implica, entre otras cosas, la construcción de la primera planta de producción en el estado de Nueva York. ${ }^{4}$

\section{DETERMINACIÓN DE LA ESTRUCTURA DE CAPITAL DE MENOR COSTO}

En este aparte se utiliza el Capital Asset Pricing Model (CAPM) y el ranking sintético de cobertura para estimación del costo de la deuda,

4 Con corte al 31 de diciembre de 2015, el anexo 1 contiene el estado de resultados resumido de la empresa y el anexo 2, el balance general. Todos los valores están en pesos colombianos; al momento de la construcción del caso, la tasa de cambio estaba, aproximadamente, 2.900 pesos por dólar. 
con el propósito de determinar el apalancamiento óptimo de acuerdo con el equilibrio entre los beneficios y costos marginales del endeudamiento. Se calcula el umbral entre los beneficios del escudo fiscal y el incremento de los costos financieros generados por una baja cobertura de intereses, que se reflejan en el incremento de las tasas exigidas por los proveedores de capital.

Algunos autores consideran que el CAPM es válido para cualquier economía; sin embargo, las características de reducido tamaño, baja liquidez e información imperfecta que se encuentran en el mercado colombiano implican la violación de los supuestos con los cuales fue concebido el modelo (costos de transacción mínimos, alta liquidez de todos los activos, acceso de inversionistas a la misma información e imposibilidad de encontrar activos infra o sobrevalorados en el mercado). Por esta razón, usamos tasas de referencia de mercados desarrollados.
Se debe mencionar que todos los valores utilizados en los cálculos en adelante fueron consultados con corte a septiembre de 2016, en las fuentes de referencia detalladas en las notas de pie de página. Se mencionan las fuentes de donde pueden ser extraídos los datos, para que los lectores puedan reconstruir la metodología; sin embargo, al hacer una nueva consulta dichos valores pueden haber sido actualizados.

\subsection{Cálculo del costo de la deuda y estimación del riesgo país}

Aquí se determina el costo de deuda al que podría acceder una empresa como AB en condiciones de mercado. Teóricamente, este corresponde a la suma de la tasa libre de riesgo ${ }^{5}$ más el margen de intermediación ${ }^{6}$ y la prima por riesgo país. El supuesto de mercados eficientes implícito en el modelo CAPM implica que se debe hacer un ajuste para su aplicación en países emergentes

5 La tasa libre de riesgo es la tasa spot de un instrumento considerado libre de riesgo como los bonos del tesoro americano. La mayoría de las bancas de inversión utilizan la duración de 10 años. Para consultar lo anterior ver http://finance.yahoo.com/bonds/compositebondrates

6 El margen de intermediación surge del costo de emisión de deuda para una firma privada en Estados Unidos y se calcula a partir de la tasa de mercado vigente asociada a la calificación de riesgo otorgada. El margen entre las tasas de mercado de bonos corporativos y la tasa libre de riesgo representa tanto costos de transacción como de intermediación, teniendo en cuenta la probabilidad de riesgo de no pago. Los márgenes asociados a cada calificación crediticia están disponibles en los rankings propuestos por las calificadoras de riesgo. En este caso usamos el ranking sintético de la página web de A. Damodaran, profesor de Stern School of business http://pages.stern.nyu. edu/ adamodar/NewHomePage/datafile/ratings.htm 
(Colombia, por ejemplo). Se debe sumar una prima por riesgo país, la cual representa el retorno adicional que exigiría un inversionista por la volatilidad adicional asociada a una economía emergente (Cosset \& Roy, 1991).

La literatura y la praxis financiera proponen diferentes métodos para calcular el riesgo país (Damodaran, 2003). Dentro de los más populares tenemos: 1) el Emerging Markets Bond Index (EMBI), diferencial entre los márgenes de los bonos colombianos con denominación en dólares y los bonos de la reserva federal de Estados Unidos; 2) el Riesgo Relativo del Capital propio, y 3) el que se inferiere de los Credit Default Swaps para Colombia. De todos los anteriores, el $\mathrm{EMBI}^{7}$ es el más practicado, pero en el presente estadio no solo utilizaremos este método, sino el de Riesgo relativo de capital propio, con el objetivo de hacer un comparativo de los resultados.
Un cuestionamiento que puede surgir al uso del EMBI radica en que la diferencia de los márgenes de los bonos del país emergente, no son suficientes para medir la exposición al riesgo de inversión en un mercado emergente. El inversionista debería esperar que el rendimiento en un mercado emergente sea mayor que el margen impuesto a la deuda soberana de dicho país, de tal forma que el riesgo país (CR) sería mejor determinado a traves del Equity Risk (ER) (Damodaran, 2015). ${ }^{7}$ Así, para saber qué tan alto debe ser este premio por riesgo país, se usa la volatilidad relativa del mercado accionario del país emergente $^{8}\left(\sigma_{\mathrm{EME}}\right)$ con la volatilidad de los rendimientos de los bonos soberanos $^{9}\left(\sigma_{\text {DSME }}\right)$ y se multiplica por el margen (spread) entre los Credit Default Swaps de la deuda de Estados Unidos $\left(\mathrm{CDS}_{\mathrm{EU}}\right)$ y los Credit Default Swaps de la deuda del país emergente $\left(\mathrm{CDS}_{\mathrm{PE}}\right)^{10}$ :

$E R=\frac{\sigma_{E M E}}{\sigma_{D S M E}} *\left(C D S_{E U}-C D S_{P E}\right)$

7 El EMBI se puede obtener de http://www.ambito.com/economia/mercados/riesgo-pais/info/?id=4

8 La desviación estándar del rendimiento del capital propio en Colombia se puede obtener de la data del Banco de la Republica. http://www.banrep.gov.co/es/igbc

9 La desviación estandar de la deuda soberana de Colombia se puede obtener de: http://www.investing.com/rates-bonds/colombia-10-year-bond-yield-historical-data

10 Los Credit Default Swaps (CDS) para Colombia y Estados unidos se pueden obtener de: http://dbresearch.com/servlet/reweb2.ReWEB? rwnode=DBRINTERNETEN-PROD\$EM\&rwobj=CDS. calias\&rwsite=DBRINTERNETEN-PROD 
La tabla 1 muestra el cálculo del riesgo país con corte al 20 de septiembre de 2016, por los dos métodos descritos. Como se puede ver, el método del Equity Risk (ER) captura una mayor volatilidad de los rendimientos de los de las acciones en Colombia.

\section{Tabla 1. Cálculo del Riesgo país}

\begin{tabular}{|l|c|}
\hline Tasa libre de riesgo (ver nota 5) & $1,53 \%$ \\
\hline Volatilidad Colcap diaria & $5,46 \%$ \\
\hline Volatilidad deuda diaria soberana Colombia & $1,82 \%$ \\
\hline Volatilidad Colcap anualizada $\left(\sigma_{\mathrm{EME}}\right)^{11}$ & $86,7 \%$ \\
\hline Volatilidad deuda soberana Colombia anualizada $\left(\sigma_{\mathrm{DSME}}\right)$ & $28,9 \%$ \\
\hline $\mathrm{CDS}$ EU $\left(C D S_{E U}\right)$ & $1,65 \%$ \\
\hline $\mathrm{CDS} \operatorname{COL}\left(C D S_{P E}\right)$ & $0,20 \%$ \\
\hline Spread $\left(C D S_{E U}-C D S_{P E}\right)$ & $1,45 \%$ \\
\hline \multicolumn{1}{|c|}{$\sigma_{E M E}$} & 3,0 \\
\hline Cociente $\sigma_{D S M E}$ & $4,35 \%$ \\
\hline riesgo país Equity Risk (ER) & $2,70 \%$ \\
\hline Riesgo país Embi & \\
\hline
\end{tabular}

Fuente: elaboración propia.

Teniendo el CR, se puede calcu- se le debe aplicar la identidad de lar el valor del costo de la deuda Fisher para ubicar la tasa en térmi$\left(\mathrm{K}_{\mathrm{d}}\right)$ para cada nivel de cobertu- nos equivalentes para Colombia. ${ }^{12}$ ra de la utilidad operativa (Ebit) Los resultados se pueden ver en la sobre los intereses; al resultado tabla 2:

11 Las volatilidades analizadas se calculan multiplicando la volatilidad diaria por la raiz de 252.

12 Se usó una tasa de inflación del 0,84\% en EU y del 7\% proyectado para Colombia. La inflación en Estados Unidos se puede obtener de http://es.global-rates.com/estadisticas-economicas/inflacion/ indice-de-precios-al-consumo/ipc/estados-unidos.aspx 
Tabla 2. Tasa de costo de la deuda

\begin{tabular}{|c|c|c|c|c|c|}
\hline \multicolumn{2}{|c|}{ Nivel de cobertura } & \multirow{2}{*}{ Calificación } & \multirow{2}{*}{ Spread } & \multirow{2}{*}{$\begin{array}{c}\mathrm{K}_{\mathrm{d}}{ }^{13} \text { local con } \\
\text { Embi }\end{array}$} & \multirow{2}{*}{$\begin{array}{c}\mathrm{K}_{\mathrm{d}} \text { local cor } \\
\text { ER }\end{array}$} \\
\hline Desde & Hasta & & & & \\
\hline 12,5 & 13,00 & Aaa/AAA & $0,75 \%$ & $11,4 \%$ & $13,1 \%$ \\
\hline 9,5 & 12,49 & $\mathrm{Aa} 2 / \mathrm{AA}$ & $1,00 \%$ & $11,7 \%$ & $13,4 \%$ \\
\hline 7,5 & 9,49 & $\mathrm{~A} 1 / \mathrm{A}+$ & $1,10 \%$ & $11,8 \%$ & $13,5 \%$ \\
\hline 6 & 7,49 & $\mathrm{~A} 2 / \mathrm{A}$ & $1,25 \%$ & $11,9 \%$ & $13,7 \%$ \\
\hline 4,5 & 5,99 & $\mathrm{~A} 3 / \mathrm{A}-$ & $1,75 \%$ & $12,5 \%$ & $14,2 \%$ \\
\hline 4 & 4,49 & $\mathrm{Baa} 2 / \mathrm{BBB}$ & $2,25 \%$ & $13,0 \%$ & $14,7 \%$ \\
\hline 3,5 & 3,99 & $\mathrm{Ba} 1 / \mathrm{BB}+$ & $3,25 \%$ & $14,1 \%$ & $15,8 \%$ \\
\hline 3 & 3,49 & $\mathrm{Ba} 2 / \mathrm{BB}$ & $4,25 \%$ & $15,1 \%$ & $16,9 \%$ \\
\hline 2,5 & 2,99 & $\mathrm{~B} 1 / \mathrm{B}+$ & $5,50 \%$ & $16,4 \%$ & $18,2 \%$ \\
\hline 2 & 2,49 & $\mathrm{~B} 2 / \mathrm{B}$ & $6,50 \%$ & $17,5 \%$ & $19,2 \%$ \\
\hline 1,5 & 1,99 & B3/B- & $7,50 \%$ & $18,6 \%$ & $20,3 \%$ \\
\hline 1,25 & 1,49 & $\mathrm{Caa} / \mathrm{CCC}$ & $9,00 \%$ & $20,2 \%$ & $21,9 \%$ \\
\hline 0,8 & 1,249 & $\mathrm{Ca} 2 / \mathrm{CC}$ & $12,00 \%$ & $23,3 \%$ & $25,1 \%$ \\
\hline 0,5 & 0,79 & $\mathrm{C} 2 / \mathrm{C}$ & $16,00 \%$ & $27,6 \%$ & $29,3 \%$ \\
\hline-10 & 0,49 & $\mathrm{D} 2 / \mathrm{D}$ & $20,00 \%$ & $31,8 \%$ & $33,6 \%$ \\
\hline
\end{tabular}

Fuente: Damodaran (2016).

\subsection{Estimación del costo del capital propio}

Para hallar el costo de capital propio se usa el modelo CAPM (Sharpe, 1964; Lintner, 1965; Mosin, 1966). El costo del capital propio $\left(\mathrm{K}_{\mathrm{E}}\right)$ es igual a la suma de la tasa libre de riesgo (RF) más la prima por riesgo (RP), multiplicado por el beta del capital propio $\left(\beta_{\mathrm{E}}\right)$ :

$K_{E}=R F+R P * \beta_{E}$

$\mathrm{RP}^{14}$ (Risk Premiun) es la diferencia promedio entre los retornos del

13 Este valor se obtuvo de: $\mathrm{Kd}$ real Embi $=[(1+1,53 \%+0,75 \%+2,7 \%) /(1+0,84 \%)]-1=4,11 \%$. Kd nominal Embi $=[(1+4,11 \%) *(1+7 \%)]-1=11,4 \%$. De manera similar se obtiene el cálculo de la Kd utilizando el ER

14 RP se calcula como el promedio aritmético de la diferencia entre los retornos del portafolio de mercado y los bonos (tbonds) del tesoro americano, desde 1928 hasta 2015. Puede ser obtenido de diferentes fuentes; en este caso, usamos la página web de del profesor de Stern School of business, A. Damodaran, http://pages.stern.nyu.edu/ adamodar/NewHomePage/datafile/histretSP.html 
mercado y la tasa libre de riesgo. Para el cálculo del beta, utilizamos el beta de empresas comparables que operen en mercados eficientes (Bowman \& Bush, 2016); esta información se puede obtener de bases de datos como Bloomberg ${ }^{\circledR}$ o Datastream ${ }^{\circledR}$. Para ser seleccionadas, las empresas comparables deben cumplir con lo siguiente: 1) estar inscritas en mercados que cumplan en el mayor grado posible la hipótesis de eficiencia, es decir, aquellos en los cuales los inversionistas pueden diversificar el riesgo no sistemático y 2) participar en el mismo sector industrial de la empresa para la cual se hace el análisis (procesamiento de alimentos), es decir, que sus ingresos provengan de un portafolio de unidades de negocio similar. Alternativamente, se pueden usar los betas sectoriales, que son consultados por muchos autores a través de sus bases de datos. ${ }^{15}$ El Beta es una medida de la exposición de la firma al riesgo sistemático o no diversificable $\mathrm{y}$, por lo tanto, es una medida de volatilidad de los rendimientos de la acción de la empresa frente a los del mercado.
3.3. Estimación de la exposición de la empresa al riesgo país (CR) y cálculo del costo de capital de la empresa desapalancada

En la estimación del costo de capital para mercados emergentes no solo es importante calcular el valor de la prima por riesgo país (CR); también se debe establecer qué tan expuesta está la empresa a ese riesgo país. Según Damodaran (2003), existen las siguientes posibilidades: la primera es suponer que todas las compañías en un país están igualmente expuestas al riesgo país; en este caso, se debe sumar la prima por riesgo país en la ecuación del cálculo del costo de capital.

$K_{E}=R F+R P * \beta_{-E}+C R$ (3)

Nosotros consideramos que la exposición de la empresa al riesgo país es proporcional a todos los otros riesgos sistemáticos, los cuales están medidos por el beta. Por tal motivo, estimamos el costo de capital sumando a la tasa libre de riesgo el producto del beta por la suma del premio de mercado y el riesgo país.

$$
K_{E}=R F+(R P+C R) * \beta_{E}
$$

15 Los betas sectoriales igualmente están contenidos en el site del profesor A. Damodaran. 


\subsection{Valor de la empresa desapalancada}

El objetivo principal de este trabajo es definir la estructura de capital y no valorar la empresa, aunque para definirla es necesario determinar el impacto de la misma en el valor de la compañía. En este caso, se supone que en los mercados maduros la utilidad económica y la tasa de crecimiento real $(\mathrm{g})$ tienden a cero. ${ }^{16}$ La inversión bruta sería financiada con la generación interna de fondos, de tal forma que el capex neto sería cero y por consiguiente el valor de la empresa sin deuda en el periodo $t$ $\left(\mathrm{V}_{\mathrm{Ut}}\right)$ se obtiene de dividir la utilidad operacional, después de impuestos del período siguiente $\left(\mathrm{NOPAT}_{\mathrm{t}+1}\right)^{17}$ sobre la tasa de costo de capital de la empresa sin deuda $\left(\mathrm{K}_{\mathrm{u}}\right)$.

$$
V_{U t}=\frac{N O P A T_{t+1}}{K_{u}}
$$

La nota técnica 1 , al final del caso contiene el desarrollo analítico de esta ecuación. ${ }^{18}$ Este cálculo se basa en la propuesta de Stern (1974), quien indica que el período de ven- taja competitiva tiende a agotarse en un horizonte de tiempo. Sin embargo, se debe tener en cuenta que en economías altamente inflacionarias, al denominador debe restarse el valor de la inflación esperada. Cuando la inflación es positiva se puede dar una estimación baja del valor real de la empresa, porque esto implicaría que el valor de los flujos de caja decrecerían a la tasa de inflación $\mathrm{y}$, por tanto, ajustes adicionales pueden ser requeridos (Bradley \& Jarrell, 2003).

El crecimiento nominal (g) mencionado en la tabla 3, solo se utiliza para proyectar el EBIT operacional al año 2016, según estimaciones que obedecen a las inversiones proyectadas. Con una inflación del 7\%, el g nominal del $30 \%$ corresponde al $21,4 \%$ real. Este crecimiento no se utiliza en el cálculo de perpetuidades por las razones expuestas antes acerca de las características de un mercado maduro y descritas con más detalle en la nota técnica 1. El supuesto es que la empresa, al estar en un mercado maduro, es incapaz de extraer rentas adicionales en el

16 En mercados maduros, el Roic tiende a hacer igual al Wacc bajo el supuesto de competencia perfecta. Cuando el Roic es igual al Wacc el crecimiento es irrelevante para el valor de la empresa.

17 Nopat=EBIT*(1-T) y T es la tasa de impuesto efectiva sin gastos no deducibles. Nopat significa utilidad operativa después de impuestos.

18 Cuando se estudie la nota técnica 1, se debe tener en cuenta que cuando la empresa no tiene deuda, el costo de capital propio $(\mathrm{Ku})$ es idéntico al costo promedio ponderado de capital (Wacc), cuyo cálculo se detalla más adelante. 
largo plazo; por tanto, su rentabilidad tiende a ser igual al costo de capital y el crecimiento es irrelevante en la generación de valor. Así, g=0 en la perpetuidad y en el periodo relevante, es decir, las ventas solo crecerían por efectos de la inflación. El lector que desee estilizar mucho más el ejercicio de valoración (no es nuestro objetivo) puede sensibilizar el modelo asumiendo distintos crecimientos en el periodo relevante (alargar el periodo de ventaja competitiva) y en la perpetuidad, teniendo en cuenta que en ese caso se debe controlar la relación entre crecimiento y reinversión. ${ }^{19}$

En la tabla 3 se pueden ver los cálculos referidos anteriormente; también se muestra el Capital invertido operativo (CIO) calculado como los activos de operación menos los proveedores de bienes y servicios y el Ebit sin gastos no recurrentes. Estos cálculos están basados en los estados financieros de la empresa.

Tabla 3. Datos operacionales y cálculo del Ku

\begin{tabular}{|c|c|c|c|}
\hline $\begin{array}{l}+ \text { Ebit } \\
+ \text { No recurrentes }\end{array}$ & $\begin{array}{l}155.034 \\
24.904\end{array}$ & $\begin{array}{l}\mathrm{RP} \\
\mathrm{Bu}\end{array}$ & $\begin{array}{l}6,18 \% \\
0,74\end{array}$ \\
\hline$=$ Ebit $_{\text {operacional }}$ & 179.938 & $\mathrm{Ku} \mathrm{EMB}^{20}$ & $8,1 \%$ \\
\hline $\mathrm{Ebit}_{\mathrm{t}+1}=\mathrm{Ebit}_{\text {operacional }} *(1+\mathrm{g})$ & 233.919 & $\mathrm{Ku}$ Equity risk & $9,32 \%$ \\
\hline UAI & 84.579 & Ku EMBI Local & $14,7 \%$ \\
\hline Impuesto & 47.671 & Ku Equity Risk local & $16,0 \%$ \\
\hline $\mathrm{T}=(\mathrm{Impuesto} / \mathrm{UAI})$ & $56,4 \%$ & g real nomina $a^{21}$ & $30 \%$ \\
\hline+ Activos & 1.015 .699 & g real esperado & $21,4 \%$ \\
\hline - Proveedores Bienes y servicios & 305.558 & Nopatt+1=Ebitt+1*(1-T) & 102.076 \\
\hline$=\mathrm{CIOt}$ & 710.141 & ROIC=Nopatt+1/CIOt & $32,94 \%$ \\
\hline
\end{tabular}

Fuente: elaboración propia.

19 El crecimiento $(\mathrm{g})$ está determinado por $\mathrm{g}=\mathrm{b} * \mathrm{ROIC}$, donde $\mathrm{b}=\mathrm{IN} / \mathrm{NOPAT}$ (inversión neta sobre Nopat); b, en la literatura es conocido como plowback ratio.

20 El Ku se refiere al costo de capital para la empresa sin deuda, considerando el riesgo país, se calcula con al ecuación (9). Por ejemplo, cuando se usa el Embi como medida del riesgo país Ku Em $\mathrm{bi}=1,53 \%+0,74(6,18 \%+2,70 \%)=8,1 \%$. Después nuevamente se usa la identidad de Fisher para llevarlo a su equivalente en términos locales (Colombia) con la inflación de Estados Unidos y la de Colombia y se llega al Ku local. De igual forma se calcula el Ku cuando se usa como medida del riesgo país el Equity Risk (Ku Equity risk)

21 El crecimiento nominal aquí mencionado solo se utiliza para proyectar el Ebit operacional al año 2016, según estimaciones de acuerdo con la inversiones proyectadas. Con una inflación del 7\%, el g nominal del $30 \%$, corresponde con un $21,4 \%$ real. Este crecimiento no se utiliza en el cálculo de perpetuidades por las razones expuestas antes acerca de las características de un mercado maduro y descritas con más detalle en la NT. 1. 
3.5. Monto de la deuda, intereses, beneficio tributario, valor del capital propio en libros, relación $D / E$ inicial y flujo de caja del accionista

Con el valor del $\mathrm{K}_{\mathrm{d}} \mathrm{y}$ los niveles de cobertura en el ranking sintético, se puede establecer el monto de la deuda correspondiente, debido a que los intereses son iguales al monto de la deuda (D) multiplicados por el $\mathrm{K}_{\mathrm{d}}$. Para hacerlo se puede usar la siguiente ecuación:

Cobertura $=\frac{\text { Ebit }}{\text { Intereses }}=\frac{\text { Ebit }}{K_{d} * D}$,

por lo tanto, $D=\frac{\text { Ebit }}{\text { Cobertura } * K_{d}}(7)$
El valor de los intereses $\left(\mathrm{K}_{\mathrm{d}}{ }^{*} \mathrm{D}\right) \mathrm{y}$ del beneficio tributario o escudo fiscal $\left(\mathrm{TS}=\mathrm{K}_{\mathrm{d}} * \mathrm{D} * \mathrm{~T}\right)$ permiten obtener el valor del capital propio, descontando al $\mathrm{K}_{\mathrm{E}}$, el flujo de caja del accionista o propietario (FCA). El FCA está determinado por el Nopat, menos el valor de los intereses, más el beneficio tributario. La relación deuda sobre capital propio inicial (D/E) se estima dividiendo el valor de la deuda (D) sobre la resta entre al CIO y el monto de la deuda. La tabla 4 muestra los resultados obtenidos; nótese que por obvias razones se obtiene el mismo valor de los intereses, TS y el FCA, sin importar la medida de riesgo país usada. En la tabla 4 solo se incluye el valor superior del rango de cobertura del ranking sintético.

Tabla 4. Monto de la deuda, intereses, beneficio tributario, valor del capital propio en libros, relación $\mathrm{D} / \mathrm{E}$ inicial y flujo de caja del accionista

\begin{tabular}{|c|c|c|c|c|c|c|c|c|c|}
\hline \multirow[t]{2}{*}{ Cobertura } & \multicolumn{2}{|c|}{$D=\frac{\text { Ebit }}{\text { Cobertura } * K_{d}}$} & \multirow[t]{2}{*}{$\begin{array}{l}\text { Intereses } \\
=\mathrm{D}^{*} \mathrm{~K}_{\mathrm{d}}\end{array}$} & \multirow{2}{*}{$\begin{array}{c}\text { Beneficio } \\
\text { tributario } \\
\mathrm{TS}=\mathrm{K}_{\mathrm{d}} * \mathrm{D} \\
* \mathrm{~T}\end{array}$} & \multicolumn{2}{|c|}{$\begin{array}{c}\text { Vr capital en libros } \\
\text { incial } \\
\mathrm{E}=\mathrm{CIO}-\mathrm{D}\end{array}$} & \multicolumn{2}{|c|}{$\mathrm{D} / \mathrm{E}$ inicial } & \multirow{2}{*}{$\begin{array}{l}\mathrm{FCA}= \\
\text { Nopat - } \\
\mathrm{D}^{* \mathrm{~K}_{\mathrm{d}}+} \\
\mathrm{TS}\end{array}$} \\
\hline & EMBI & ER & & & EMBI & ER & EMBI & ER & \\
\hline sin deuda & - & - & - & - & $710.141,0$ & $710.141,0$ & 0 & 0 & $102.076,1$ \\
\hline 13,00 & $121.432,5$ & $105.282,5$ & $13.841,4$ & $7.801,4$ & $588.708,5$ & $604.858,5$ & 0,206 & 0,174 & $96.036,1$ \\
\hline 12,49 & $123.516,2$ & $107.414,1$ & $14.406,6$ & $8.119,9$ & $586.624,8$ & $602.726,9$ & 0,211 & 0,178 & $95.789,5$ \\
\hline 9,49 & $161.096,8$ & $140.260,3$ & $18.960,8$ & $10.686,8$ & $549.044,2$ & $569.880,7$ & 0,293 & 0,246 & $93.802,1$ \\
\hline 7,49 & $201.389,7$ & $175.644,9$ & $24.023,8$ & $13.540,4$ & $508.751,3$ & $534.496,1$ & 0,396 & 0,329 & $91.592,8$ \\
\hline 5,99 & $241.097,8$ & $211.427,8$ & $30.039,7$ & $16.931,2$ & $469.043,2$ & $498.713,2$ & 0,514 & 0,424 & $88.967,6$ \\
\hline 4,49 & $308.505,5$ & $271.906,9$ & $40.075,3$ & $22.587,5$ & $401.635,5$ & $438.234,1$ & 0,768 & 0,620 & $84.588,4$ \\
\hline 3,99 & $320.947,8$ & $285.430,2$ & $45.097,2$ & $25.418,0$ & $389.193,2$ & $424.710,8$ & 0,825 & 0,672 & $82.396,9$ \\
\hline
\end{tabular}




\begin{tabular}{|c|c|c|c|c|c|c|c|c|c|}
\hline \multirow[t]{2}{*}{ Cobertura } & \multicolumn{2}{|c|}{$D=\frac{\text { Ebit }}{\text { Cobertura } * K_{d}}$} & \multirow{2}{*}{$\begin{array}{l}\text { Intereses } \\
=\mathrm{D}^{*} \mathrm{~K}_{\mathrm{d}}\end{array}$} & \multirow{2}{*}{$\begin{array}{c}\text { Beneficio } \\
\text { tributario } \\
\mathrm{TS}=\mathrm{K}_{\mathrm{d}}{ }^{* \mathrm{D}} \\
* \mathrm{~T}\end{array}$} & \multicolumn{2}{|c|}{$\begin{array}{c}\text { Vr capital en libros } \\
\text { incial } \\
\mathrm{E}=\mathrm{CIO}-\mathrm{D}\end{array}$} & \multicolumn{2}{|c|}{$\mathrm{D} / \mathrm{E}$ inicial } & \multirow{2}{*}{$\begin{array}{c}\mathrm{FCA}= \\
\text { Nopat - } \\
\mathrm{D}^{* \mathrm{~K}_{\mathrm{d}}+} \\
\mathrm{TS}\end{array}$} \\
\hline & EMBI & ER & & & EMBI & ER & EMBI & ER & \\
\hline 3,49 & $341.164,4$ & $305.785,7$ & $51.558,2$ & $29.059,6$ & $368.976,6$ & $404.355,3$ & 0,925 & 0,756 & $79.577,5$ \\
\hline 2,99 & $366.083,9$ & $330.889,7$ & $60.179,9$ & $33.919,0$ & $344.057,1$ & $379.251,3$ & 1,064 & 0,872 & $75.815,2$ \\
\hline 2,49 & $412.939,2$ & $375.429,0$ & $72.264,3$ & $40.730,1$ & $297.201,8$ & $334.712,0$ & 1,389 & 1,122 & $70.542,0$ \\
\hline 1,99 & $487.153,4$ & $445.213,9$ & $90.421,1$ & $50.963,8$ & $222.987,6$ & $264.927,1$ & 2,185 & 1,681 & $62.618,8$ \\
\hline 1,49 & $599.239,9$ & $551.400,1$ & $120.763,8$ & $68.065,7$ & $110.901,1$ & $158.740,9$ & 5,403 & 3,474 & $49.378,1$ \\
\hline 1,25 & $617.347,3$ & $574.316,5$ & $144.065,7$ & $81.199,3$ & $92.793,7$ & $135.824,5$ & 6,653 & 4,228 & $39.209,8$ \\
\hline 0,79 & $825.826,7$ & $776.594,9$ & $227.769,6$ & $128.377,1$ & $-115.685,7$ & $-66.453,9$ & $-7,139$ & $-11,686$ & $2.683,6$ \\
\hline 0,49 & $.153 .861,0$ & $1.093 .769,8$ & $367.220,4$ & $206.975,3$ & $-443.720,0$ & $-383.628,8$ & $-2,600$ & $-2,851$ & $-58.169,0$ \\
\hline
\end{tabular}

Fuente: elaboración propia.

3.6. Ajuste del Beta por apalancamiento, cálculo del KE y del valor del capital propio a precios de mercado

Como sabemos, el apalancamiento incrementa el nivel de rendimiento exigido por los inversionistas $\left(\mathrm{K}_{\mathrm{E}}\right)$, ante el incremento de la posibilidad de default y los costos de estrés financiero; por lo tanto, se cumple que $K_{E}>K_{U}$. La forma de llegar al $\mathrm{K}_{\mathrm{E}}$ es apalancando el $\beta_{\mathrm{U}}$ por el nivel de $\mathrm{D} / \mathrm{E}$ seleccionada para obtener un $\beta_{E}$ (Fernandez, 2006). La siguiente es la ecuación más utilizada para apalancar y desapalancar los betas (Hamada, 1972):

$\beta_{E}=\beta_{U}\left(1+(1-T) \frac{D}{E}\right)$

Sin embargo, este es un resultado especial que aplica solo cuando el nivel de deuda neta es constante y cuando el escudo fiscal como la deuda neta se consideran libres de riesgo. De hecho, aunque esta ecuación es comúnmente usada, no es correcta para ajustar las diferencias por apalancamiento cuando los niveles de deuda neta sobre el valor de la empresa cambian en el tiempo. En particular, no aplica para el caso que necesitamos aquí, pues la empresa debe mantener un ratio constante de deuda neta sobre los flujos de caja descontados al Wacc. Para este caso, Miles y Ezzell (1985) mencionan que cuando la firma ajusta su deuda neta en el tiempo a una proporción fija de su valor, el nivel de deuda neta está perfectamente correlacionado con el valor de la firma. Así, el escudo fiscal tiene el mismo riesgo que el valor de la firma y, por lo tanto, 
el mismo beta. De esta forma, el beta apalancado podría calcularse como:

$\beta_{E}=\beta_{U}\left(1+\left(1-t * k_{d}\left(1+k_{d}\right)^{-1}\right)(\mathrm{D} / \mathrm{E})\right)$

Donde $t$ es la tasa de impuestos. Estos autores asumen deuda sin riesgo porque cuando el apalancamiento se ajusta al final de cada periodo, el flujo de caja del primer periodo es libre de riesgo. En este caso, el beta de los activos apalancados es el promedio ponderado de $\beta_{\mathrm{U}}$ y el beta del escudo fiscal libre de riesgo $\left(\beta_{\mathrm{TS}}\right)$, el cual es cero. Si suponemos ajustes continuos de la estructura de capital para maximizar el valor de la empresa, entonces el $\beta_{D}$ tiende a cero y esta última ecuación puede ser usada. En la nota técnica 2 se puede ver el desarrollo analítico que sustenta esta conclusión.

Debido a que los supuestos para el uso del CAPM se basan en valores de mercado de la deuda y el capital propio, debemos implementar un cálculo iterativo o la referencia circular de la hoja electrónica, a partir del D/E inicial para llegar al valor del capital propio a precios de mercado. Con este resultado se puede apalancar el Beta con la relación D/E a valores de mercado. El cálculo iterativo se inicia apalancando $\beta_{U}$ con la relación $D / E$ inicial para cada nivel de apalancamiento en el ranking y así obtener un primer $\mathrm{K}_{\mathrm{E}}$ que se lleva a términos nominales en Colombia para obtener el valor del capital propio a precios de mercado usando el FCA, es decir $\mathrm{V}_{\mathrm{E}}=\mathrm{FCA} / \mathrm{K}_{\mathrm{E}}$. El proceso se repite recalculando el $\mathrm{D} / \mathrm{E}$ con el nuevo valor del capital propio, hasta que el $\mathrm{K}_{\mathrm{E}} \mathrm{y}$ el capital propio concurran a un valor estable. Después de aproximadamente 10 iteraciones para cada nivel de endeudamiento, los valores tienen una convergencia a los que se ven en la tabla 5 .

Tabla 5. Cálculo del costo del capital propio $\left(\mathrm{K}_{\mathrm{E}}\right)$, El valor del capital propio a precios de mercado $\left(V_{E}\right)$ y el Valor de la empresa $\left(V_{0}\right)$ con distintos niveles de cobertura

\begin{tabular}{|c|c|c|c|c|c|c|c|c|c|c|}
\hline \multicolumn{2}{|c|}{ Cobertura } & \multirow{3}{*}{ Calificación } & \multicolumn{2}{|c|}{ Deuda } & \multicolumn{2}{|c|}{$\mathrm{KE}$} & \multicolumn{2}{|c|}{ VE } & \multicolumn{2}{|c|}{ Vo } \\
\hline Desde & Hasta & & EMBI & ER & EMBI & ER & EMBI & ER & EMBI & ER \\
\hline $\begin{array}{c}\text { Sin } \\
\text { deuda }\end{array}$ & $\begin{array}{c}\text { Sin } \\
\text { deuda }\end{array}$ & & - & - & $14,7 \%$ & $16,0 \%$ & $693.902,1$ & $637.803,6$ & $693.902,1$ & $637.803,6$ \\
\hline 12,5 & 13,00 & $\mathrm{Aaa} / \mathrm{AAA}$ & $121.432,5$ & $105.282,5$ & $16,0 \%$ & $15,8 \%$ & $598.601,7$ & $606.205,7$ & $720.034,2$ & $711.488,2$ \\
\hline 9,5 & 12,49 & $\mathrm{Aa} 2 / \mathrm{AA}$ & $123.516,2$ & $107.414,1$ & $16,1 \%$ & $15,9 \%$ & $596.064,8$ & $603.644,3$ & $719.580,9$ & $711.058,3$ \\
\hline 7,5 & 9,49 & $\mathrm{~A} 1 / \mathrm{A}+$ & $161.096,8$ & $140.260,3$ & $16,6 \%$ & $16,3 \%$ & $565.826,6$ & $575.633,6$ & $726.923,4$ & $715.893,9$ \\
\hline
\end{tabular}




\begin{tabular}{|c|c|c|c|c|c|c|c|c|c|c|}
\hline \multirow{2}{*}{ Cobertura } & \multirow{2}{*}{ Calificación } & \multicolumn{2}{|c|}{ Deuda } & \multicolumn{2}{c|}{ KE } & \multicolumn{2}{c|}{ VE } & \multicolumn{2}{c|}{ Vo } \\
\cline { 5 - 9 } Desde & Hasta & & EMBI & ER & EMBI & ER & EMBI & ER & EMBI & ER \\
\hline 6 & 7,49 & A2/A & $201.389,7$ & $175.644,9$ & $17,2 \%$ & $16,8 \%$ & $532.910,4$ & $545.025,6$ & $734.300,1$ & $720.670,5$ \\
\hline 4,5 & 5,99 & A3/A- & $241.097,8$ & $211.427,8$ & $17,9 \%$ & $17,4 \%$ & $497.644,5$ & $511.599,2$ & $738.742,3$ & $723.027,0$ \\
\hline 4 & 4,49 & Baa2/BBB & $308.505,5$ & $271.906,9$ & $19,3 \%$ & $18,6 \%$ & $438.262,1$ & $455.466,0$ & $746.767,6$ & $727.372,9$ \\
\hline 3,5 & 3,99 & Ba1/BB+ & $320.947,8$ & $285.430,2$ & $19,7 \%$ & $18,9 \%$ & $418.555,3$ & $435.231,6$ & $739.503,1$ & $720.661,8$ \\
\hline 3 & 3,49 & Ba2/BB & $341.164,4$ & $305.785,7$ & $20,3 \%$ & $19,5 \%$ & $391.208,7$ & $407.799,7$ & $732.373,1$ & $713.585,4$ \\
\hline 2,5 & 2,99 & B1/B+ & $366.083,9$ & $330.889,7$ & $21,3 \%$ & $20,4 \%$ & $355.662,7$ & $372.141,0$ & $721.746,6$ & $703.030,7$ \\
\hline 2 & 2,49 & B2/B & $412.939,2$ & $375.429,0$ & $23,5 \%$ & $22,2 \%$ & $300.228,5$ & $317.768,0$ & $713.167,7$ & $693.197,1$ \\
\hline 1,5 & 1,99 & B3/B- & $487.153,4$ & $445.213,9$ & $29,1 \%$ & $26,7 \%$ & $215.136,9$ & $234.721,4$ & $702.290,3$ & $679.935,3$ \\
\hline 1,25 & 1,49 & Caa/CCC & $599.239,9$ & $551.400,1$ & $61,9 \%$ & $48,7 \%$ & $79.717,0$ & $101.493,5$ & $678.956,8$ & $652.893,6$ \\
\hline 0,8 & 1,249 & Ca2/CC & $617.347,3$ & $574.316,5$ & $181,3 \%$ & $113,4 \%$ & $21.630,0$ & $34.591,1$ & $638.977,3$ & $608.907,7$ \\
\hline
\end{tabular}

Fuente: elaboración propia.

En la tabla 5, el valor de la empresa apalancada $\left(\mathrm{V}_{\mathrm{o}}\right)$ para los diversos niveles de cobertura sa obtiene de sumar el valor del capital propio a precios de mercado $\left(\mathrm{V}_{\mathrm{E}}\right)$ más el valor de la deuda $\left(V_{\mathrm{o}}=\mathrm{V}_{\mathrm{E}}+\mathrm{D}=\mathrm{E}+\mathrm{D}\right)$. El cálculo se hizo utilizando ambos indicadores de riesgo país (EMBI y ER); nótese que el mayor valor de la empresa coincide para el mismo nivel de cobertura, entre 4 y 4,49 veces.

\subsection{Valor de la empresa apalancada mediante el cálculo del Wacc}

El valor de la empresa apalancada se determina por dos caminos. El primero suma el valor del capital propio a los precios de mercado y el saldo de la deuda; el segundo calcula el costo promedio pondera- do de capital para descontar el Nopat $_{\mathrm{t}+1}$, tal como se mencionó antes $\left(\mathrm{V}_{\mathrm{o}}=\right.$ Nopat $_{\mathrm{t}+1} /$ Wacc $)$. El Wacc se calcula como el promedio ponderado del costo de la deuda después de impuestos y del capital propio para cada nivel de endeudamiento:

$W a c c=K_{d}(1-T)\left(\frac{D}{D+E}\right)+K_{E}\left(\frac{E}{D+E}\right)$

En la tabla 6 se puede observar que el $V_{o}$ calculado con el Nopat ${ }_{t+1} y$ el Wacc da el mismo valor que el calculado antes, sumando la deuda más el capital propio a precios de mercado. Además, se denota que la estructura de capital óptima (D/E) minimiza el Wacc y, a su vez, maximiza el valor de la empresa, sin importar la medida de CR usada. Sin embargo, dado que la prima por riesgo país es mayor por el ER, 
la relación $\mathrm{D} / \mathrm{E}$ óptima es más baja primidos por parsimonia y el costo usando este método. Algunos valo- de la deuda está expresado después res del extremo inferior fueron su- de impuestos $\left(\mathrm{K}_{\mathrm{d}}{ }^{*}\right)$.

Tabla 6. Cálculo de la estructura de capital que minimiza el Wacc y maximiza el Valor de la empresa (VO)

\begin{tabular}{|c|c|c|c|c|c|c|c|c|c|c|c|c|}
\hline \multicolumn{2}{|c|}{ Cobertura } & \multirow{2}{*}{ Calificación } & \multicolumn{5}{|c|}{ Con $\mathrm{CR}=\mathrm{EMBI}$} & \multicolumn{5}{|c|}{ Con $\mathrm{CR}=\mathrm{ER}$} \\
\hline Desde & Hasta & & $\mathrm{D} / \mathrm{E}$ & $\mathrm{K}_{\mathrm{D}}^{* 22}$ & $\mathrm{KE}$ & WACC & Vo & $\mathrm{D} / \mathrm{E}$ & $\mathrm{K}_{\mathrm{D}}^{*}$ & $\mathrm{KE}$ & WACC & Vo \\
\hline $\begin{array}{c}\text { Sin } \\
\text { deuda }\end{array}$ & $\begin{array}{c}\text { Sin } \\
\text { deuda }\end{array}$ & - & - & - & $14,7 \%$ & $14,7 \%$ & $693.902,1$ & - & - & $16,0 \%$ & $16,0 \%$ & $637.803,6$ \\
\hline 12,5 & 13,00 & Aaa/AAA & 0,21 & $4,97 \%$ & $16,0 \%$ & $14,2 \%$ & $720.034,2$ & 0,17 & $5,74 \%$ & $15,8 \%$ & $14,3 \%$ & $711.488,2$ \\
\hline 9,5 & 12,49 & $\mathrm{Aa} 2 / \mathrm{AA}$ & 0,21 & $5,09 \%$ & $16,1 \%$ & $14,2 \%$ & $719.580,9$ & 0,18 & $5,85 \%$ & $15,9 \%$ & $14,4 \%$ & $711.058,3$ \\
\hline 7,5 & 9,49 & $\mathrm{~A} 1 / \mathrm{A}+$ & 0,29 & $5,14 \%$ & $16,6 \%$ & $14,0 \%$ & $726.923,4$ & 0,25 & $5,90 \%$ & $16,3 \%$ & $14,3 \%$ & $715.893,9$ \\
\hline 6 & 7,49 & $\mathrm{~A} 2 / \mathrm{A}$ & 0,40 & $5,21 \%$ & $17,2 \%$ & $13,9 \%$ & $734.300,1$ & 0,33 & $5,97 \%$ & $16,8 \%$ & $14,2 \%$ & $720.670,5$ \\
\hline 4,5 & 5,99 & $\mathrm{~A} 3 / \mathrm{A}-$ & 0,51 & $5,44 \%$ & $17,9 \%$ & $13,8 \%$ & $738.742,3$ & 0,42 & $6,20 \%$ & $17,4 \%$ & $14,1 \%$ & $723.027,0$ \\
\hline 4 & 4,49 & $\mathrm{Baa} 2 / \mathrm{BBB}$ & 0,77 & $5,67 \%$ & $19,3 \%$ & $13,7 \%$ & $746.767,6$ & 0,62 & $6,43 \%$ & $18,6 \%$ & $14,0 \%$ & $727.372,9$ \\
\hline 3,5 & 3,99 & $\mathrm{Ba} 1 / \mathrm{BB}+$ & 0,82 & $6,13 \%$ & $19,7 \%$ & $13,8 \%$ & $739.503,1$ & 0,67 & $6,89 \%$ & $18,9 \%$ & $14,2 \%$ & $720.661,8$ \\
\hline 3 & 3,49 & $\mathrm{Ba} 2 / \mathrm{BB}$ & 0,92 & $6,59 \%$ & $20,3 \%$ & $13,9 \%$ & $732.373,1$ & 0,76 & $7,36 \%$ & $19,5 \%$ & $14,3 \%$ & $713.585,4$ \\
\hline 2,5 & 2,99 & $\mathrm{~B} 1 / \mathrm{B}+$ & 1,06 & $7,17 \%$ & $21,3 \%$ & $14,1 \%$ & $721.746,6$ & 0,87 & $7,94 \%$ & $20,4 \%$ & $14,5 \%$ & $703.030,7$ \\
\hline 2 & 2,49 & $\mathrm{~B} 2 / \mathrm{B}$ & 1,39 & $7,64 \%$ & $23,5 \%$ & $14,3 \%$ & $713.167,7$ & 1,12 & $8,40 \%$ & $22,2 \%$ & $14,7 \%$ & $693.197,1$ \\
\hline 1,5 & 1,99 & B3/B- & 2,18 & $8,10 \%$ & $29,1 \%$ & $14,5 \%$ & $702.290,3$ & 1,68 & $8,86 \%$ & $26,7 \%$ & $15,0 \%$ & $679.935,3$ \\
\hline
\end{tabular}

Fuente: elaboración propia.

Se pueden ver ambos resultados en las figuras 1 y 2 para $C R=E M B I$ y para $\mathrm{CR}=\mathrm{ER}$.

$22 \mathrm{KD}^{*}=\mathrm{KD} *(1-\mathrm{T})$, donde $\mathrm{T}$ es la tasa de impuestos. 
Figura 1. Estructura óptima de capital con $\mathrm{CR}=\mathrm{EMBI}$

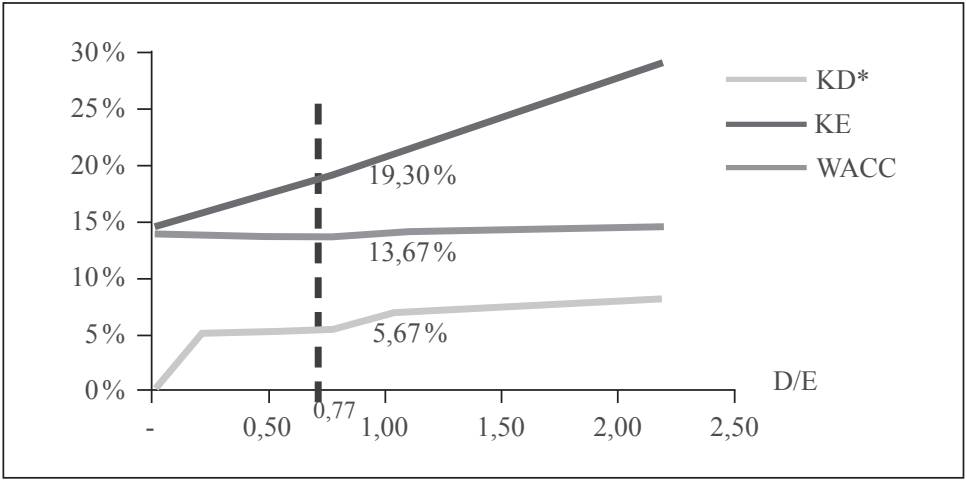

Fuente: elaboración propia.

\section{ANÁLISIS DE RESULTADOS}

Con los cálculos realizados hasta este punto, se procede a comparar la estructura óptima con la que posee actualmente la empresa. También se contrasta el costo teórico de acceso a la deuda con el que se está pagando realmente y se determina el costo del capital propio que deberían estar cargando los socios a la empresa por concepto de uso del capital propio, de acuerdo con la estructura actual de endeudamiento. Finalmente, se hace una análisis de la equivalencia entre los fundamentales y el precio de las acciones preferenciales transadas recientemente. El análisis de resultados se cierra haciendo una propuesta de restructuración y se indica cuál sería el valor generado por la adopción de la estructura propuesta.

\subsection{Comparación del costo de deuda actual y teórico ${ }^{23}$}

La tabla 7 muestra el detalle de las deudas actuales de la empresa, sin

23 Nótese que el cálculo del valor Kd real de la empresa no se hace con el ánimo de calcular el Wacc actual de la empresa, ni con la intensión de valorar la empresa con base en su costo de capital actual. Se hace solo con el fin de comparar el costo de deuda proveniente de la calificación del ranking sintético, versus el costo de la deuda real de la empresa. Nuestra intención no es hacer una valoración de la empresa, pero, si así fuera, hacerlo con el Wacc actual podría constituir un error, en la medida que quienes adquieren pueden refinanciar la deuda a precios de mercado. Pero si se insiste en ello debería usarse un cálculo del Wacc que considere los ahorros tributarios por devaluación (ver Södersten, 1982). El objetivo del caso es hallar la estructura de capital que minimice el Wacc, por ello en los cálculos de valoración usamos el $\mathrm{Kd}$ al cual los inversionistas podrían conseguir en el mercado local, la deuda para esta empresa, sin consideraciones de parenting, ni mercados internos de capital. En ese sentido no asumimos deuda en moneda extranjera en el cálculo de la estructura de capital. 
considerar un sobregiro temporal que está a una tasa del 30\% EA. Se calcula el promedio ponderado de las deudas en moneda local y extranjera, considerando la devaluación/revaluación corrida desde el momento de la firma de los contratos. En la parte inferior se muestra el valor teórico del ranking sintético, que indica cuánto debe costar la deuda de la empresa; este se obtiene después de calcular la cobertura actual que tiene la compañía con el EBIT que genera y los intereses que está pagando. Para la fecha de realización de estos cálculos, la calificación crediticia según el ranking sintético para su nivel de cobertura seria B3/B- y el costo correspondiente el $18.6 \%$ (en la tabla 2 el valor esta entre el 18,6\% y el $20,3 \%$ ), el cual es muy similar al $18,26 \%$ que se está pagando actualmente. ${ }^{24}$

Tabla 7. Comparación entre el Kd teórico y Real

\begin{tabular}{|c|c|c|c|c|c|c|}
\hline Fuente & Saldo & Costos & $\%$ & $\mathrm{Kd} \sin \mathrm{DEV}^{25}$ & $\mathrm{Kd}$ con $\mathrm{DEV}$ & Ponderado \\
\hline \multicolumn{7}{|l|}{ Bonos } \\
\hline Serie 1 & 121.920 & $\mathrm{IPC}+6,7 \%$ & $22,9 \%$ & $13,70 \%$ & $13,70 \%$ & $3,14 \%$ \\
\hline serie 2 & 51.580 & $11,49 \%$ & $9,7 \%$ & $11,49 \%$ & $11,49 \%$ & $1,11 \%$ \\
\hline Serie 3 & 86.500 & $\mathrm{IPC}+7,4 \%$ & $16,3 \%$ & $14,40 \%$ & $14,40 \%$ & $2,34 \%$ \\
\hline \multicolumn{7}{|l|}{ Deuda } \\
\hline Bancolombia 1 & 45.457 & Libor $180+3,720$ & $8,5 \%$ & $10,1 \%$ & $28,9 \%$ & $2,47 \%$ \\
\hline Bancolombia 2 & 29.296 & Libor $180+3,720$ & $5,5 \%$ & $10,1 \%$ & $24,4 \%$ & $1,34 \%$ \\
\hline BBVA1 & 83.448 & Libor $180+2,624$ & $15,7 \%$ & $7,84 \%$ & $20,7 \%$ & $3,25 \%$ \\
\hline BBVA2 & 65.832 & Libor $180+2,648$ & $12,4 \%$ & $7,89 \%$ & $25,8 \%$ & $3,19 \%$ \\
\hline BanBogota 1 & 5.016 & Libor $180+1,270$ & $0,9 \%$ & $5,05 \%$ & $4,8 \%$ & $0,05 \%$ \\
\hline BanBogota 2 & 17.323 & Libor $180+1,320$ & $3,3 \%$ & $5,15 \%$ & $5,4 \%$ & $0,18 \%$ \\
\hline Leasing & 25.395 & $24,89 \%$ & $4,8 \%$ & $24,89 \%$ & $24,9 \%$ & $1,19 \%$ \\
\hline Total & 531.767 & & $100,0 \%$ & & Kd real & $18,26 \%$ \\
\hline Interés pagado & 97.110 & & & & & \\
\hline Ebit & 179.938 & & & & & \\
\hline Cobertura & 1,85 & & & & & \\
\hline Kd teórico & $18,56 \%$ & & & & & \\
\hline
\end{tabular}

Fuente: elaboración propia.

24 El $\mathrm{K}_{\mathrm{d}}$ se determina usando el EMBI como CR, se deja al lector para que lo haga usando el ER. La tasa de leasing anunciada en las notas de los estados financieros es 1,02\% NMV; sin embargo, una mejor estimación puede sacarse de http://www.grupobancolombia.com/wps/portal/personas/ productos-servicios/leasing/vehiculo/simulador-credito-vehiculo/\#\#resultado

La libor a 180 se puede obtener de http://indicadoreseconomicos.bccr.fi.cr/indicadoreseconomi$\cos /$ Cuadros/frmVerCatCuadro.aspx?idioma $=1 \&$ CodCuadro $=\% 20342$

El histórico de la tasa de cambio para calcular la devaluación se puede obtener http://www.banrep.gov.co/es/trm

25 La sigla DEV se refiere a la devaluación calculada para cada crédito en dólares, que están suscritos a una tasa compuesta por libor más puntos adicionales. Por ejemplo, para la deuda denominada Bancolombia1, se tomó la cotización del dólar en el momento del desembolso (1926,83 pesos) y la tasa de cambio al momento de la realización del caso (2924,89 pesos), se calculó la tasa efectiva 
4.2. KE de la estructura actual y crecimiento implícito en la última transacción de venta de acciones privilegiadas

Al 31 de diciembre de 2015, el capital social de la empresa estaba compuesto por un capital autorizado de 18.000 millones de pesos, representado en 180 millones de acciones, con un valor nominal de 100 pesos cada una, las cuales podrían ser emitidas en distintas clases (ordinarias o privilegiadas). Las acciones en circulación a diciembre 31 de 2015 eran de 156.586.992, pero en la asamblea extraordinaria de accionistas del 12 de febrero de 2015 se aprobó la emisión de acciones privilegiadas de valor nominal de 100 pesos cada una con una prima en colocación de 2.777,46 pesos por acción.
Con fines meramente académicos, podemos hacer un análisis de la operación a la luz de los fundamentales de la empresa y determinar cuál es la tasa de crecimiento implícito asociada al valor de la prima. Para tales efectos, calculamos el FCA restando del Nopat el valor de los intereses pagados y sumando el beneficio tributario. Se usa la misma técnica del cálculo interactivo presentada anteriormente para apalancar el $\beta_{U}$, empezando con una relación $\mathrm{D} / \mathrm{E}$ calculada con el saldo de la deuda actual y el capital propio resultante de la restarle al CIO el valor de tal deuda. Usando el ER como medida de CR y después de 15 iteraciones el $\mathrm{K}_{\mathrm{E}}$ converje a $33,98 \%$ y el valor del capital propio pasa a $175,707 \mathrm{mi}-$ llones de pesos, tal como se observa en la tabla 8 .

Tabla 8. Cálculo del KE y el crecimiento implícito en prima de la acción privilegiada

\begin{tabular}{|l|c|}
\hline FCA & 59.700 \\
\hline $\mathrm{K}_{\mathrm{E}}$ & $33,98 \%$ \\
\hline $\mathrm{E}=\mathrm{V}_{\mathrm{E}}$ (valor del capital propio según fundamentales) & 175.707 \\
\hline Relación D/E con valores de mercado según fundamentales & 3,026 \\
\hline $\mathrm{V}_{\mathrm{o}}=\mathrm{D}+\mathrm{E}$ (valor empresa apalancada=deuda y capital propio) & 707.474 \\
\hline Número de acciones en circulación & 156.589 .992 \\
\hline Valor acción última transacción & 2.877 \\
\hline Valor del capital propio según última transacción & 450.581 \\
\hline Crecimiento implícito & $18,30 \%$ \\
\hline Wacc & $14,43 \%$ \\
\hline $\mathrm{V}_{\mathrm{o}}$ (valor de la empresa apalancada usando el Wacc) & 707.474 \\
\hline
\end{tabular}

Fuente: elaboración propia.

anual de devaluación (17,1\%) para el periodo transcurrido (2,65 años), por tanto la tasa efectiva para amortización semestral sería: libor $180+3,720=(1+(1,22344 \%+3,72 \%)) 2-1=10,1 \%$. Después de la devaluación: $(1+10,1 \%) *(1+17,1 \%)-1=28,9 \%$. 
Puede notarse que el $\mathrm{K}_{\mathrm{E}}$ sube de manera considerable para ese nivel de cobertura. Eso ocurre debido a la alta relación $\mathrm{D} / \mathrm{E}$ resultante de la estructura actual $(3,03)$, que indica que tiene tres veces más deuda que valor de mercado del capital propio. Existe también una diferencia considerable entre el valor del capital propio según los fundamentales y el que resulta de multiplicar el número de acciones circulantes por el valor de la acción transada en la emisión reciente de acciones privilegiadas. El $\mathrm{K}_{\mathrm{E}}$ y el $\mathrm{K}_{\mathrm{d}}$ de la estructura de capital actual nos permiten establecer el Wacc de la empresa y volver a establecer el valor de la empresa apalancada $\left(\mathrm{V}_{\mathrm{o}}\right)$ utilizando el Wacc para descontar el Nopat ${ }_{t+1}$. Como en la ocasión anterior, este último coincide con el valor de suma de la deuda y el capital propio a precios de mercado.

La diferencia hallada entre los valores del capital propio según nuestros cálculos y el resultante de la transacción de las acciones privilegiadas debería corresponder al crecimiento esperado posterior a $\mathrm{t}+1$, de acuerdo con las proyecciones de la empresa. Ese crecimiento implícito (g) se puede obtener despejándolo de la ecuación para cálculo del valor a continuidad:

$g=\frac{E^{*} K_{E}-F C A}{F C A+E}$

El valor obtenido para el $g$ implícito (ver tabla 8) indica un crecimiento futuro a perpetuidad del $18,3 \%$, con una inflación proyectada del $4,5 \%$ el crecimiento real sería del $13,2 \%{ }^{26}$

\subsection{Propuesta de recapitalización}

En la tabla 9, se muestra una propuesta de recapitalización para acceder a la estructura óptima de capital hallada. Se cambiarían 223.261 millones de pesos de deuda por capital propio obtenido de una nueva emisión. Sin el crecimiento implícito serían de 162,5 millones de acciones, pero si se mantiene la prima en colocación anterior, serían de 77,6 millones de acciones. Con ello se incrementaría el valor de la empresa apalancada en

26 Es evidente que pueden existir muchos determinantes por los cuales en la mayoría de las transacciones el precio de mercado no coincide con el valor modelado en una valoración de empresas, sobre todo en los proceso de fusiones y adquisiciones (ver por ejemplo: Roll, 1986; Berkman, Bradbury \& Ferguson, 2000; Lai \& Wong, 2015). La aproximación mas conocida en este sentido es asociada con la cita del famoso inversionista Warren Buffet: "Price is what you pay, value is what you get." Obviamente en los precios de una transacción influyen factores asociados al estado del mercado en el momento que se hace la transacción y el valor relativo de la firma para quien compra. El ejercicio que se hace en este caso es solo con fines académicos y no pretende establecer un juicio sobre el valor transado. 
cerca de 40.000 millones de pesos. Nótese que el valor de la acción con la estructura propuesta se incremen- ta en un $22,4 \%$ frente al valor que resulta de valorar la empresa con la estructura actual de endeudamiento.

Tabla 9. Propuesta de reestructuración (valores en millones pesos)

\begin{tabular}{|l|c|c|c|}
\hline \multirow{2}{*}{ Estructura } & \multirow{2}{*}{ Actual } & \multicolumn{2}{c|}{ Propuesta } \\
\cline { 3 - 4 } & & Sin g implícito & Con g implícito \\
\hline Deuda (millones) & 531.767 & 308.506 & \\
\hline Vr capital propio (millones) & 175.707 & 438.262 & \\
\hline V+E & 707.474 & 746.768 & \\
\hline D/E & 3,03 & 0,70 & \\
\hline Acciones(millones) & 156,5 & 319,0 & \\
\hline Valor acción & $1.122,73$ & $1.373,81$ & 2877,46 \\
\hline Deuda a disminuir (millones) & & 223.261 & 223.261 \\
\hline Número de acciones nuevas a emitir (millones) & & 162,5 & 77,6 \\
\hline
\end{tabular}

Fuente: elaboración propia.

\section{CONCLUSIONES}

Cómo las empresas determinan su estructura de capital ha sido un fenómeno ampliamente estudiado por diversos autores. Muchos académicos se han enfocado en establecer el óptimo ratio de endeudamiento que maximiza el valor de la empresa, considerando los distintos costos asociados a la deuda y al capital propio. Otros tantos han tratado de explicar cómo hacen las empresas al momento de tomar decisiones sobre el financiamiento de las inversiones y cuáles son los criterios que los gerentes usan para seleccionar y acceder a diferentes fuentes de financiamiento. No obstante, el tema de la estructura de capital se considera un problema sin resolver en la literatura financiera.

Por lo tanto, es entendible que estudiosos de temas financieros y gerentes de empresas se muestren confundidos acerca de cómo funcionan todos los postulados teóricos acerca de este tema y que cuestionen la practicidad o utilidad de muchos conceptos. El desarrollo de este caso se centró en aplicar a una empresa particular, en un mercado emergente, algunos de los conceptos asociados a la teoría de la estructura de capital, para conciliarlos y aterrizarlos a resultados concretos en una empresa real. 
Para ello, se usaron varias técnicas que permitieron aplicar tres principios, que fueron establecidos para empresas en mercados desarrollados y para una empresa del sector de alimentos en el mercado emergente colombiano. Además Se utilizaron el Capital Asset Pricing Model (CAPM) y diversos cálculos del riesgo país, así como las propuestas de Miles y Ezzell (1985) para apalancar el beta en condiciones de deuda sobre patrimonio constante a precios de mercado. Finalmente, se realizó un cálculo recursivo a partir del flujo de caja del accionista para determinar el costo del capital propio a precios de mercado. Todo lo anterior se hizo teniendo en cuenta que la acción de la empresa seleccionada tiene una muy baja liquidez.

Posteriormente, se usó el ranking sintético de cobertura para estimación del costo de la deuda, para determinar el apalancamiento óptimo determinado por el equilibrio entre los beneficios y costos marginales del endeudamiento. Se presentó el umbral entre los beneficios del escudo fiscal y los posibles costos de insolvencia financiera generados por una baja cobertura de intereses, que se reflejan en el incremento de las tasas exigidas por los proveedores de capital.

Los resultados muestran cómo en la medida que aumenta el endeu- damiento se incrementa el riesgo y por tanto hay mayor retorno requerido por parte de los inversionistas. Además, se comprueba que la estructura de capital óptima maximiza el valor de la firma y el precio de la participación de cada socio (acciones o participación societaria), a la vez que minimiza el costo promedio ponderado del capital. El aprendizaje resultante de este trabajo, además de ser una ayuda de orden práctico para la toma de decisiones de endeudamiento, constituye la base para continuar avanzando en la aplicación de los conceptos teórico-prácticos relacionados con la fijación de estructuras de capital para empresas en mercados emergentes. Quienes estén interesados en el tema pueden utilizar este trabajo como base para avanzar en la aplicación práctica de otros elementos teóricos, por ejemplo, aquellos relacionados con ¿cuál otra teoría de estructura de capital podría ser aplicada en este caso (pecking order, costos de agencia)?, ¿qué otro método podría usarse para apalancar los betas?, ¿cuáles serían las ventajas y desventajas de cada método?, ¿cómo cambia el crecimiento implícito esperado de la empresa según la transacción de acciones preferenciales realizadas recientemente, si se opta por ampliar el periodo de ventaja competitiva, es decir, se utiliza un crecimiento para periodo relevante de 5 años en el proceso de valoración?, si se hace 
el ajuste anterior, ¿cómo cambia la estructura optima de capital y qué beneficios puede generar?, ¿cómo cambia la propuesta de reestructuración si se considera el disponible para calcular la deuda neta?

\section{REFERENCIAS}

Almeida, H., \& Philippon, T. (2008). Estimating Risk-Adjusted Costs of Financial Distress, Journal of Applied Corporate Finance, 20(4), 110-114.

Baker, M., \& Wurgler, J. (2002). Market Timing and Capital Structure. Journal of Finance, LVII(1), 1-32.

Barclay, M., Smith, C., \& Watts, R. (1995). The Determinants of Corporate Leverage and Dividend Policies, Journal of Applied Corporate Finance, 7, 4-19.

Berkman, H., Bradbury, M., \& Ferguson, J. (2000). The Accuracy of Price-Earnings and Discounted Cash Flow Methods of IPO Equity Valuation. Journal of International Financial Managing and Accounting, 11(2), 71-83.

Bowman, R., \& Bush, S. (2016). Using Comparable Companies to Estimate the Betas of Private Companies. Journal of Applied Finance, Forthcoming. Recuperado de https://ssrn.com/abstract $=956443$

Bradley, M., \& Jarrell, G. (2003). Inflation and the Constant-Growth
Valuation Model. A Clarification. Recuperado de http://ssrn. com $/$ abstract $=356540$

Brennan, M.J., \& Schwartz, E.S. (1984).Optimal Financial Policy and Firm Valuation. The Journal of Finance, 39(3), 593-607.

Bris, A., Welch, I., \& Zhu, N. (2006). The Costs of Bankruptcy: Chapter 7 Liquidation versus Chapter 11 Reorganization. The Journal of Finance, 51(3), 1253-1303.

Cosset, J., \& Roy, J. (1991). The Determinants of Country Risk Ratings. International Bussines Studies, 22(1), 135-142.

Damodaran, A. (2003). Measuring Company Exposure to Country Risk: Theory and Practice. Recuperado de https://ssm.com/ abstract $=889388$

Damodaran,A. (2015), Country Risk: Determinants, Measures and Implications. Recuperado de http:// ssrn.com/abstract $=2630871$

Fernandez, P. (2006). Levered and Unlevered Beta, Working paper No. 488. IESE Business School. Universidad de Navarra.

Glover, B. (2016). The Expected Cost of Default. Journal of Financial Economics, 119(2), 284-299.

Graham, J. R. (2000). How Big Are the Tax Benefits of Debt? Journal of Finance, 55, 1901-41.

Graham, J. R. (2003). Taxes and Corporate Finance: A Review. Review of Financial Studies, 16 , 1074-1129. 
Graham, J. R., \& Leary, M. T. (2011). A Review of Empirical Capital Structure Research and Directions for the Future. Annual Review of Financial Economics, 3, 309-345.

Hamada, R. (1972) The Effect of the Firm's Capital Structure on the Systematic Risk of Common Stock. The Journal of Finance, 27, 435-452.

Harris, M., \& Raviv, A. (1991). The Theory of Capital Structure, Journal of Finance, 46(1), 297355.

Hakkio, C., \& Keeton, W. (2009). Financial Stress: What Is It, How Can It Be Measured, and Why Does It Matter? Economic Review, 94(2), 5-50.

Hovakimian, A., Opler, T., \& Titman, S. (2001). The Debt-Equity Choice. Journal of Financial and Quantitative Analysis, 36, 1-24.

Lai, D., \& Wong, W.(2015). An Empirical Study of relationship between share price and intrinsic value of companies. Financial Studies, 4, 65-89.

Lian, Y. (2017). Financial Distress and Customer-Supplier Relationships. Journal of Corporate Finance, 43: 397-406.

Lintner, J. (1965). The Valuation of Risk Assets and the Selection of Risky Investments in Stock Portfolios and Capital Budgets.
Review of Economics and Statistics, 47(1), 13-37.

Jensen, M., \& Meckling, W. (1976): Theory of the Firm: Managerial Behavior, Agency Cost and Ownership Structure. Journal of Financial Economics, 3, 305-360. Jensen, M. (1986). Agency Costs of Free Cash Flow, Corporate Finance, and Takeovers, American Economic Review, 76(2), 323-329.

Leland, H. (1998). Agency Costs, Risk Management, and Capital Structure. Journal of Finance, 49, 1213-43.

Miles, J., \& Ezzell, R. (1985). Reformulating Tax Shield Valuation: A note. Journal of Finance, 40, 1485-1492.

Modigiani, F., \& Miller, M. (1958). The Cost of Capital, Corporation Finance and the Theory of Investment. American Economic Review, 48(3), 261-297.

Miller, M. (1977). Debt and Taxes. Journal of Finance, 32(2): 261275.

Mosin, J. (1966). Equilibrium in a Capital Asset Market. Econometrica, 34(4): 768-783.

Myers, S. (1984). The Capital Structure Puzzle. Journal of Finance, 39(3), 575-591.

Myers, S. (1977). Determinants of Corporate Borrowing, Journal of Financial Economics, 5(2), 147175. 
Myers S., \& Majluf N. (1984). Corporate Financing and Investment Decisions When Firms Have Information that Investors Do Not Have. Journal of Financial Economics, 13, 187-221.

Parsons, C., \& Titman, S. (2008). Empirical Capital Structure: A Review. Foundations and Trends in Finance, 3(1), 1-93.

Popescu, L., \& Visinescu, S. (2009). A Review of Capital Structure Theories. Annals of Faculty of Economics, 3(1), 315-320.

Rajan, R. G., \& Zingales, L. (1995). What Do We Know About Capital Structure? Some Evidence from International Data. Journal of Finance, 50(5), 1421-1460.

Roll, R. (1986), The Hubris Hypothesis of Corporate Takeovers. Journal of Business, 59(2), 197216.

Ross, S. (1977). The Determination of Financial Structure: the Incentive-Signalling Approach. Bell Journal of Economics, 8(1), 24-40.

Sharpe, W. (1964). A Theory of Market Equilibrium under Conditions of Risk. Journal of Finance, 19(3), 425-442.
Shelifer, A., \& Vihsny, R. (1992). Liquidation Values and Debt Capacity: A Market Equilibrium Approach, Journal of Finance, 47, 1343-1366.

Smith, C.(1986). Investment Banking and the Capital Acquisition Process. Journal of Financial Economics, 15, 3-29.

Södersten, J. (1982) Accelerated Depreciation and the Cost of Capital. Scandinavian Journal of Economics, 84(1), 111-115.

Stern, J. (1974). Earnings Per Share Don't Count. Financial Analysts Journal, 30(4), 39-75.

Titman, S. (1984). The Effect of Capital Structure on a Firm's Liquidation Decision. Journal of Financial Economics, 13, 137151.

Titman, S., \& Wessels, R. (1988). The Determinants of Capital Structure Choice. Journal of Finance, 43,1-19.

Vasiliou, D., \& Daskalakis, N. (2009). Institutional Characteristics and Capital Structure: A Cross-National Comparison. Global Finance Journal, 19(3): 286-306. 


\section{NOTA TÉCNICA 1}

Stern (1974) definió el tiempo de horizonte o de ventaja competitiva (H) aquel en donde Roic $^{27}>$ Wacc, después de $\mathrm{H}$, la empresa pierde la ventaja competitiva y por tanto

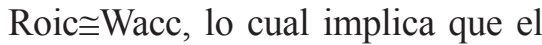
crecimiento no crea valor y es irrelevante. En valoración de empresas, $(\mathrm{H})$ indica cuando termina el periodo relevante. A partir de allí, el valor de continuidad (CV) puede ser calculado descontando el flujo de caja libre (FCF) proyectado un periodo después del periodo relevante, a la tasa de crecimiento real $\left(\mathrm{g}_{\mathrm{R}}\right)$ con el Wacc y el Roic en términos reales $\left(\right.$ Wacc $_{R}$ y Roic ${ }_{R}$ ) con una inflación " $i$ ”. Partimos de:

$C V=\frac{F C F_{t}(1+g)}{\text { Wacc-g }}$

Teniendo en cuenta que:

Wacc- $g=\left(1+\right.$ Wacc $\left._{R}\right)(1+i)-1-\left(1+g_{R}\right)$

$(1+i)+1$

Wacc-g $=(1+\mathrm{i})\left(1+\right.$ Wacc $\left._{R}-1-g_{R}\right)$

Wacc-g $=(1+\mathrm{i})\left(\right.$ Wacc $\left._{R}-g_{R}\right)$

Por otra parte, el plowback ratio (b) o grado de reinversión está determi- nado por el cociente entre la inversión neta $\left(\mathrm{IN}^{28}\right)$ y la utilidad operativa neta de impuestos (Nopat).

$b=\frac{I N}{\text { Nopat }}$

La tasa de crecimiento sostenible en términos reales $\left(\mathrm{g}_{\mathrm{R}}\right)$ se expresa como:

$g_{R}=b^{*}$ Roic $_{R}$

Por tanto el flujo de caja libre sería:

$F C F_{t}=(1-b)$ Nopat $_{t}$

$F C F_{t}=\left(1-\frac{g_{R}}{\text { Roic }_{R}}\right)$ Nopat $_{t}$

De allí que el valor de continuidad quedaría:

$C V=\frac{\left(1-\frac{g_{R}}{\text { Wacc }_{R}}\right) \text { Nopat }{ }^{t+1}}{\left(\text { Wacc }_{R}-g_{R}\right)(1+i)}$

$C V=\frac{\left(\frac{\text { Wacc }_{R}-g_{R}}{\text { Wacc }_{R}}\right) \text { Nopat }{ }^{++1}}{\left(\text { Wacc }_{R}-g_{R}\right)(1+i)}$

$C V=\frac{\text { Nopat }_{t+1}}{\left(\frac{\text { Wacc }-i}{1+i}\right)(1+i)}$

$C V=\frac{\text { Nopat }_{t+1}}{\text { Wacc }-i}$

27 Roic $=$ Ebit $_{t+1} *(1-\mathrm{t}) / \mathrm{Cio}_{\mathrm{t}}=\mathrm{Nopat}_{\mathrm{t}+1} / \mathrm{Cio}_{\mathrm{t}}$, donde $t$ es la tasa de impuestos.

$28 \mathrm{IN}=\mathrm{KTNO}+\mathrm{CAPEX}$-Depreciación; $\mathrm{KTNO}=$ Activos corrientes operacionales - Pasivos corrientes operacionales. 
Por tanto, para calcular el valor de la empresa a perpetuidad en un mercado maduro donde Roic $\cong$ Wacc se usa la ecuación 23, que supone $\mathrm{IN}=0 \quad$ (capex $=$ Generación interna de Fondos) y no crecimiento. Para nuestro caso usamos $i \cong 0$ y el nopat en $\mathrm{t}+1$ se aproximó al flujo de caja de bruto menos la variación del capital de trabajo proyectada a $\mathrm{t}+1$.

\section{NOTA TÉCNICA 2}

La necesidad de apalancar y desapalancar el beta surge de:

$\beta_{A}=\frac{D}{D+E} \beta_{D}+\frac{E}{D+E} \beta_{E}=d \beta_{D}+(1-d) \beta_{E}$

$\beta_{A}=\left(1+\frac{D}{\mathrm{E}}\right) \beta_{A}-\frac{D}{\mathrm{E}} \beta_{D}$

Es decir, el riesgo sistemático del capital propio $\left(\beta_{\mathrm{E}}\right)$ se incrementa con el endeudamiento siempre que se cumpla que el beta del activo $\left(\beta_{\mathrm{A}}\right)$ sea mayor que el beta de la deuda $\left(\beta_{\mathrm{D}}\right)$. Cuando la estructura de capital es óptima o con deuda libre de riesgo, el beta de la deuda tiende a cero, por tanto, el beta del capital propio queda solo en función del beta del activo. Bajo impuestos corporativos el valor a precio de mercado puede re-expresarse como la suma del valor presente de los flujos de caja sin deuda $\left(\mathrm{V}_{\mathrm{U}}\right)$ más el valor presente del escudo fiscal $\left(\mathrm{V}_{\mathrm{TS}}\right)$ :
$V_{A}=D+E=V_{U}+V_{T S}$

Por lo tanto el beta de los activos podría calcularse como:

$\beta_{A}=\frac{D+E-V_{T S}}{D+E} \beta_{U}+\frac{V_{T S}}{D+E} \beta_{T S}$

Donde $\beta_{\mathrm{U}}$ es el beta asociado a los flujos de caja de la empresa sin deuda y $\beta_{\text {TS }}$. Es el beta asociado al escudo fiscal. Hamada (1972) considera que debido a que el escudo fiscal (TS) es proporcional a la deuda neta, se puede asumir que el beta de la deuda es similar al beta del escudo fiscal y si la deuda es constante y libre de riesgo entonces tanto el beta de la deuda como el del escudo fiscal serían cero. Por tanto, el valor presente del escudo fiscal sería el producto de la tasa de impuestos $(\mathrm{T})$, la tasa de interés $\left(\mathrm{K}_{\mathrm{d}}\right)$ y la deuda (D) descontado a la tasa de la deuda; por eso, al reemplazar el beta del activo y derivar el beta del capital propio tendríamos:

$V_{T S}=\frac{T^{*} K_{d}^{*} D}{K_{d}}=T^{*} D$, si $\beta_{D}=\beta_{T S}=0$,

entonces

$\beta_{A}=\frac{D+E-T^{*} D}{D+E} \beta_{U}$

$\beta_{U} \frac{D+E-T^{*} D}{D+E}=\frac{E}{D+E} \beta_{E}$

$B_{E}=B_{U}\left(\frac{D+E-T^{*} D}{E}\right)=B_{U}\left(\frac{D}{E}+1-\mathrm{T} * \frac{\square}{E}\right)$ 
$B_{E}=B_{U}\left(1+(1-\mathrm{T}) \frac{D}{E}\right)$

Sin embargo, esta solución solo aplica para deuda constante en términos de su valor en libros y escudo fiscal libre de riesgo. En nuestro caso, esperamos que la empresa mantenga una tasa constante de endeudamiento frente a su valor del mercado.
Miles y Ezzell (1985) anotan que en este caso el procedimiento para desapalancar el beta debe considerar que el escudo fiscal después del primer periodo es incierto y que tiene el mismo riesgo de los flujos de caja que provienen de los activos. Entonces, el valor de la empresa se puede expresar como:

$$
\begin{aligned}
& V_{A}=\sum_{J}^{T}=1 \frac{F C F_{U}}{\left(1+K_{U}\right)^{J}}+\frac{t^{*} K_{d}^{*} d^{*} V_{A}}{\left(1+K_{d}\right)}+\sum_{J}^{T}=2 \frac{T S_{J}}{\left(1+\mathrm{K}_{\mathrm{U}}\right)^{\mathrm{J}-1}\left(1+\mathrm{k}_{\mathrm{d}}\right)} \\
& V_{A}=\frac{F C F_{U}{ }^{l}+V_{U}{ }^{l}}{\left(1+K_{U}\right)}+\frac{t^{*} K_{d}^{*} d^{*} V_{A}}{\left(1+K_{d}\right)}+\frac{V_{T S}{ }^{l}}{\left(1+\mathrm{K}_{U}\right)}
\end{aligned}
$$

En la ecuación anterior, el valor de la empresa (activos) con deuda está expresado en tres componentes: el primero es el valor esperado de la empresa sin deuda, el segundo es el valor con certeza del escudo fiscal del primer periodo, y el tercero es el valor esperado del valor presente del escudo fiscal sin considerar el primer periodo.

$\beta_{A}$ puede ser expresado como el promedio ponderado de los coeficientes beta asociados a estos tres términos. Se había mencionado que los términos uno y tres tienen el mismo riesgo y, por tanto la misma tasa de descuento; luego, el beta asociado a estos dos es $\beta_{U}$ y, dado que la tasa de descuento del segundo componente es la tasa libre de riesgo, entonces su beta es cero. El valor de mercado del segundo componente sería: $=\frac{t^{*} K_{d} * d(D+E)}{\left(1+k_{d}\right)}$

y por ende la fraccion $V_{A}$ que tiene un $\beta_{U}$ de seria

$=\frac{t^{*} K_{d}{ }^{*} d}{1+k_{d}}$ y entonces $\left(1-\frac{t^{*} K_{d}{ }^{*} d}{1+k_{d}}\right) \beta_{U}$

Con deuda sin riesgo, tenemos que:

$\beta_{A}=(1-d) \beta_{E}$

$\left(1-\frac{t^{*} K_{d}^{*} d}{1+k_{d}}\right) \beta_{U}=(1-d) \beta_{E}$

$\left(\frac{1-t * k_{d}^{*} d\left(1+k_{d}\right)^{-1}}{1-d}\right) \beta_{U}=\beta_{E}$

$\left(\frac{1-t * k_{d}^{*} d\left(1+k_{d}\right)^{-1}\left(\frac{D / E}{1+D / E}\right)}{1-\frac{D / E}{1+D / E}}\right) \beta_{U}=\beta_{E}$ 
$\left(1+D / E-t^{*} k_{d}\left(1+k_{d}\right)^{-1}(D / E)\right)$

$\beta_{U}=\beta_{E}$

$\left(1+\left(1-t^{*} k_{d}\left(1+k_{d}\right)^{-1}(D / E)\right)\right.$

$\beta_{U}=\beta_{E}$

La anterior es la ecuación que hemos usado para apalancar la beta en este caso. Sin embargo, si $k_{d}$ es

(41) pequeño y con los continuos ajustes de $(D / E)$ disminuye, entonces esta expresión tiende a:

$(1+(D / E)) \beta_{U}=\beta_{E}$

Que es la usada en muchos textos. 


\section{ANEXO 1. ESTADO DE RESULTADOS}

\section{PRODUCTOS ALIMENTICIOS AB S.A.}

Estados de Resultados Integrales

Años terminados el

31 de diciembre de

$$
20152014
$$

(expresados en millones de pesos)

Ingresos de actividades ordinarias

$\$ 1.625 .894$

$\$ 1.506 .196$

Costo de ventas

Ganancia bruta

Gastos de administración y venta

Gastos de distribución

Gastos por beneficios a los empleados

Otros gastos

Otros ingresos

Resultados de actividades de la operación

Participación en la utilidad pérdida neta de subsidiarias

Ingresos financieros

$-\$ 958.496$

Costos financieros

Costo financiero, neto

Utilidad antes de impuesto a las ganancias

Gasto por impuesto a las ganancias

Resultado neto del año

Otros resultados integrales

Cambios en el valor razonable de coberturas del flujo de efectivo

Pérdidas actuariales por planes de beneficios definidos

Ganancias en conversión para operaciones extranjeras

Variaciones patrimoniales método de participación

Impuesto a las ganancias sobre otro resultado integral

Otro resultado integral total neto del año

Resultado integral total neto del año

Utilidad neta por acción (en pesos)
$\$ 667.398$

$-\$ 289.630$

$-\$ 47.732$

$-\$ 157.910$

-\$24.904

$\$ 7.812$

$\$ 155.034$

$\$ 2.455$

$\$ 11.431$

$-\$ 896.267$

$\$ 609.929$

$-\$ 301.988$

-\$44.166

$-\$ 146.414$

$-\$ 15.790$

$\$ 84.341$

$-\$ 72.910$

$\$ 84.579$

$\$ 47.447$

-\$47.671

-\$31.213

$\$ 36.908$

$\$ 16.234$

\begin{tabular}{cc}
\hline \hline$\$ 540$ & $-\$ 167$ \\
$-\$ 162$ & $-\$ 41$ \\
$\$ 10.828$ & $\$ 4.124$ \\
$\$ 16.886$ & $\$ 5.993$ \\
$\$ 210$ & $\$ 65$ \\
\hline$\$ 27.222$ & $\$ 9.974$ \\
\hline$\$ 64.130$ & $\$ 26.208$ \\
\hline \hline 235,71 & 103,70
\end{tabular}




\title{
ANEXO 2. BALANCE GENERAL
}

\author{
PRODUCTOS ALIMENTICIOS AB S.A. \\ Estados separados de Situación Financiera
}

\section{ACTIVOS}

Activos corrientes

Efectivo y equivalentes de efectivo

Cuentas por cobrar

Inventarios

Gastos pagados por anticipado

Instrumentos financieros derivados

Otros impuestos recuperables

Otros activos

Total activos corrientes

Activos no corrientes

Cuentas por cobrar

Inventarios

Inversiones

Otros activos

Propiedad, planta y equipo

Total activos no corrientes

\section{TOTAL ACTIVOS}

\section{PATRIMONIO Y PASIVOS}

\section{Al 31 de diciembre de}

Al 1 de enero de

Pasivos

Pasivos corrientes

Obligaciones financieras

Cuentas por pagar

Otras provisiones

Instrumentos financieros derivados

Otros pasivos financieros corrientes

Otros pasivos corrientes

Total pasivos corrientes

Pasivos no corrientes

Obligaciones financiera

Cuentas por pagar

Otros pasivos

Títulos emitidos

Total pasivos no corrientes

TOTAL PASIVOS

\section{PATRIMONIO}

Capital suscrito y pagado

Prima de emisión

Reservas

Otro resultado integral

Ganancias acumuladas

TOTAL PATRIMONIO

TOTAL PASIVO Y PATRIMONIO
2015

2014

(Expresados en millones de pesos)

\begin{tabular}{ccc}
$\$ 99.334,00$ & $\$ 62.095,00$ & $\$ 78.757,00$ \\
$\$ 115.161,00$ & $\$ 87.595,00$ & $\$ 79.247,00$ \\
$\$ 115.340,00$ & $\$ 112.878,00$ & $\$ 116.015,00$ \\
$\$ 4.803,00$ & $\$ 1.735,00$ & $\$ 2.881,00$ \\
$\$ 243,00$ & $\$-$ & $\$ 62,00$ \\
$\$ 3.052,00$ & $\$ 4.179,00$ & $\$ 21.356,00$ \\
$\$ 296,00$ & $\$ 1.088,00$ & $\$ 3.363,00$ \\
\hline$\$ 338.229,00$ & $\$ 269.570,00$ & $\$ 301.681,00$ \\
\hline & & \\
$\$ 133.339,00$ & $\$ 79.256,00$ & $\$ 38.985,00$ \\
$\$ 3.727,00$ & $\$ 3.200,00$ & $\$ 2.996,00$ \\
$\$ 77.038,00$ & $\$ 56.986,00$ & $\$ 49.174,00$ \\
$\$ 12.946,00$ & $\$ 17.841,00$ & $\$ 29.165,00$ \\
$\$ 450.420,00$ & $\$ 436.903,00$ & $\$ 457.463,00$ \\
\hline$\$ 677.470,00$ & $\$ 594.186,00$ & $\$ 577.783,00$ \\
\hline$\$ 1.015 .699,00$ & $\$ 863.756,00$ & $\$ 879.464,00$ \\
\hline
\end{tabular}

\section{4}

\begin{tabular}{ccc}
$\$ 28.046,00$ & $\$ 24.451,00$ & $\$ 41.037,00$ \\
$\$ 180.949,00$ & $\$ 137.796,00$ & $\$ 140.706,00$ \\
$\$ 8.456,00$ & $\$ 3.766,00$ & $\$ 3.971,00$ \\
$\$ 783,00$ & $\$ 167,00$ & $\$ 93,00$ \\
$\$ 842,00$ & $\$ 559,00$ & $\$ 497,00$ \\
$\$ 78.049,00$ & $\$ 62.259,00$ & $\$ 46.683,00$ \\
\hline$\$ 297.125,00$ & $\$ 228.998,00$ & $\$ 232.987,00$ \\
\hline & & \\
$\$ 244.247,00$ & $\$ 175.963,00$ & $\$ 177.956,00$ \\
$\$ 7.658,00$ & $\$ 5.395,00$ & $\$ 5.284,00$ \\
$\$ 28.821,00$ & $\$ 31.751,00$ & $\$ 38.276,00$ \\
$\$ 264.889,00$ & $\$ 263.909,00$ & $\$ 263.426,00$ \\
\hline$\$ 545.615,00$ & $\$ 477.018,00$ & $\$ 484.942,00$ \\
\hline$\$ 842.740,00$ & $\$ 706.016,00$ & $\$ 717.929,00$ \\
& & \\
$\$ 15.659,00$ & $\$ 15.656,00$ & $\$ 15.656,00$ \\
$\$ 43.389,00$ & $\$ 43.305,00$ & $\$ 43.305,00$ \\
$\$ 28.500,00$ & $\$ 24.563,00$ & $\$ 20.676,00$ \\
$\$ 27.222,00$ & $\$ 9.974,00$ & $\$-$ \\
$\$ 58.189,00$ & $\$ 64.242,00$ & $\$ 81.898,00$ \\
\hline$\$ 172.959,00$ & $\$ 157.740,00$ & $\$ 161.535,00$ \\
\hline$\$ 1.015 .699,00$ & $\$ 863.756,00$ & $\$ 879.464,00$ \\
\hline & &
\end{tabular}

